\title{
Multiple growth-correlated life history traits estimated simultaneously in individuals
}

\author{
Fabian Mollet ${ }^{1,2, *}$, Bruno Ernande ${ }^{3,2}$, Thomas Brunel ${ }^{1,3}$, Adriaan D. Rijnsdorp $^{1,4}$ \\ ${ }^{1}$ Wageningen IMARES, Inst. for Marine Resources and Ecological Studies, PO Box 68, NL-1970 AB IJmuiden, \\ the Netherlands \\ ${ }^{2}$ Evolution and Ecology Program, International Institute for Applied System Analysis IIASA, Schlossplatz 1, A- \\ 2361 Laxenburg, Austria \\ ${ }^{3}$ IFREMER, Laboratoire Ressources Halieutiques, Avenue du Général de Gaulle, BP32, F-14520 Port-en-Bessin, \\ France. \\ 4 Aquaculture and Fisheries group, Wageningen University, P.O.Box 338, NL-6700 AH Wageningen, The \\ Netherlands
}

*: Corresponding author : F. Mollet, email address : Fabian.Mollet@wur.nl

\begin{abstract}
:
We present a new methodology to estimate rates of energy acquisition, maintenance, reproductive investment and the onset of maturation (four-trait estimation) by fitting an energy allocation model to individual growth trajectories. The accuracy and precision of the method is evaluated on simulated growth trajectories. In the deterministic case, all life history parameters are well estimated with negligible bias over realistic parameter ranges. Adding environmental variability reduces precision, causes the maintenance and reproductive investment to be confounded with a negative error correlation, and tends, if strong, to result in an underestimation of the energy acquisition and maintenance and an overestimation of the age and size at the onset of maturation. Assuming a priori incorrect allometric scaling exponents also leads to a general but fairly predictable bias. To avoid confounding in applications we propose to assume a constant maintenance (three-trait estimation), which can be obtained by fitting reproductive investment simultaneously to size at age on population data. The results become qualitatively more robust but the improvement of the estimate of the onset of maturation is not significant. When applied to growth curves back-calculated from otoliths of female North Sea plaice Pleuronectes platessa, the four-trait and three-trait estimation produced estimates for the onset of maturation very similar to those obtained by direct observation. The correlations between life-history traits match expectations. We discuss the potential of the methodology in studies of the ecology and evolution of life history parameters in wild populations.
\end{abstract}


42 The schedule according to which energy is allocated to either somatic growth or 43 reproduction is a cornerstone of life history theory (Kooijman 1986, Roff 1992, Stearns 44 1992, Kozlowski 1996, Charnov, et al. 2001). Energy allocation schedules differ among 45 species as they reflect adaptation to both the environment and internal constraints 46 resulting from sharing a common currency between different functions. Individuals 47 indeed face an energy trade-off between somatic growth and reproduction (Roff 1992, 48 Stearns 1992, Heino and Kaitala 1999). In case of indeterminate growth, individuals also 49 experience a trade-off between current and future reproduction since fecundity generally 50 increases with body size. Various energy allocation schedules have been proposed in the 51 literature (Von Bertalanffy and Pirozynski 1952, Day and Taylor 1997, Kooijman 2000, 52 West, et al. 2001). They differ mostly in terms of priorities of energy flows to the 53 different functions. Allocation schedules typically comprise four traits, namely energy 54 acquisition, maintenance, onset of maturation, and thereafter reproductive investment, 55 whereas somatic growth arises as a by-product: the energy that remains after accounting 56 for the primary energy flows to maintenance and reproductive investment is available for 57 somatic growth. The study of energy allocation schedules in individual organisms is 58 difficult because of a lack of data at the individual level as this would require monitoring 59 separate individual organisms throughout their life time. Studies therefore have focused 60 on the population level as well as on single traits (Stevenson and Woods Jr. 2006).

61 Studying the four traits together (acquisition, maintenance, onset of maturation and 
62 reproductive investment) at the individual level would offer several advantages over the

63 widely used single trait estimation at the population level: (1) phenotypic correlations

64 between traits could be estimated; (2) changes in one trait could be interpreted

65 conditionally on changes in other traits, precisely because of the previous correlations;

66 (3) it would be more consistent with the fact that physiological trade-offs apply at the

67 individual and not at the population level.

68 Organisms in which the individual growth history is recorded in hard structures offer a 69 unique opportunity to study energy allocation schedules at the individual level. Fish for 70 instance show indeterminate growth and the growth history of individuals can be 71 reconstructed from the width of the seasonal structures imprinted in hard structures such 72 as otoliths or scales (Runnström 1936, Rijnsdorp, et al. 1990, Francis and Horn 1997).

73 Earlier studies have attempted to estimate the onset of maturation using growth history 74 reconstructed from otoliths or scales (Rijnsdorp and Storbeck 1995, Engelhard, et al. 75 2003, Baulier and Heino 2008), but no study has yet attempted to simultaneously 76 estimate several life history traits related to life time patterns of energy allocation at the 77 individual level.

78 In this study, we estimate simultaneously parameter values at the individual level for 79 energy acquisition, maintenance, onset of reproduction, and reproductive investment by 80 fitting an energy allocation model to individual growth trajectories. The energy allocation 81 model assumes that the onset of maturation is reflected in a discontinuity in the slope of 82 the growth trajectory, while the energy acquisition discounted by maintenance is assessed 83 by the slope of the growth trajectory before maturation, and reproductive investment is 84 translated in the amplitude of the change in the slope of growth trajectory at the 
85 discontinuity. The performance of the method and its sensitivity to both model 86 uncertainty and inter-annual environmental variability are explored using simulated data. 87 The method is applied to an empirical data set of individual growth curves back88 calculated from otoliths of female North Sea plaice (Pleuronectes platessa). Maturity 89 status deduced from the age and size at the onset of maturation estimated by our model is 90 compared to direct evaluation of maturity status by visual inspection of the gonads in 91 market sampling (Grift, et al. 2003). 
94 Energy allocation model. When an animal becomes mature, a proportion of the

95 available energy is channeled towards reproduction and is no longer available for somatic

96 growth (Ware 1982). Hence, a decrease in growth rate can be expected after maturation.

97 We use a general energy allocation model (Von Bertalanffy and Pirozynski 1952,

98 Charnov, et al. 2001, West, et al. 2001, Banavar, et al. 2002) according to which the

99 growth rate of juveniles and adults is given by

$100 \frac{\partial w}{\partial t}=\left\{\begin{array}{lll}a w^{\alpha}-b w^{\beta} & \text { if } t<t_{\text {mat }} \\ & 102 \\ a w^{\alpha}-b w^{\beta}-c w^{\gamma} & \text { if } \quad t \geq t_{\text {mat }}\end{array}\right.$

103 where $w$ is body weight, $t$ is time, $t_{\text {mat }}$ is time at the onset of maturation, $a w^{\alpha}$ is the rate

104 of energy acquisition, $b w^{\beta}$ is the rate with which energy is spent for maintenance and

$105 c w^{\gamma}$ is the rate of reproductive investment with which energy is spent for reproductive

106 activity (e.g. gamete production, reproductive behavior). For simplicity we will refer to

107 energy acquisition $a$, maintenance $b$ and reproductive investment $c$, although $a, b$ and

$108 c$ describe the size-specific rates for the corresponding processes. There is disagreement

109 about the scaling exponents $\alpha, \beta$, and $\gamma$ involved in the allometries between energy

110 rates and body weight. Metabolic theory of ecology (MTE) suggests that metabolism 
111 scales with a quarter power law of body weight (West, et al. 1999, Gillooly, et al. 2001,

112 Savage, et al. 2004). This hypothesis builds on the fractal-like branching pattern of

113 distribution networks involved in energy transport (West, et al. 1997) but the generality

114 of this allometric scaling law is contested (Banavar, et al. 2002, Darveau, et al. 2002,

115 Clarke 2004, Kozlowski and Konarzewski 2004). Nevertheless, we assumed a scaling

116 exponent of energy acquisition $\alpha=3 / 4$ (West, et al. 1997) as this is close to empirical

117 estimates of $\alpha$ (Gillooly, et al. 2001, Brown, et al. 2004) including our model species

118 North Sea plaice (Fonds, et al. 1992). For the scaling exponent of maintenance $\beta$, it is

119 required that $\beta>\alpha$ in order to obtain (i) bounded asymptotic growth, i.e. to reach an

120 asymptotic maximum body weight in the absence of maturation and (ii) an energetic

121 reproductive-somatic index (RSI), defined as the ratio of reproductive investment over

122 body weight in terms of energy (in other terms an energetic analogue to the gonado-

123 somatic index), that increases with age and size as commonly observed in empirical data

124 (not shown). MTE suggests $\beta=1$ since with increasing size, the energy demand becomes

125 relatively more important than its supply (West, et al. 1997, West, et al. 2001) and thus

126 fulfills the required conditions. For the scaling exponent of reproductive investment $\gamma$,

127 we assume $\gamma=1$ for the sake of simplicity. This is in line with the assumption that total

128 brood mass is a constant fraction of maternal body weight (Blueweiss, et al. 1978,

129 Charnov, et al. 2001), although reproductive investment might be related to a body

130 weight allometry with an exponent higher than 1 (Roff 1991).

131 By integration of Eq. (1) assuming $\alpha=3 / 4$ and $\beta=\gamma=1$, the somatic weight $w$ can be 132 expressed as a function of time $t$. To switch from juvenile $\left(t<t_{\text {mat }}\right)$ to adult $\left(t \geq t_{\text {mat }}\right)$ 
133 growth in Eq. (1), a continuous logistic switch function $S(t)$ with an inflection point

134 located at the time of the onset of maturation $t_{\text {mat }}$ is used (Appendix A1). It results that 135 the lifespan somatic growth curve is obtained as a continuous function of time though a 136 discontinuity in its parameters due to the onset of maturation being introduced by the 137 switch function $S(t)$ : $w^{1-\alpha}(t)=(1-S(t))\left[\frac{a}{b}-\left(\frac{a}{b}-w_{0}^{1-\alpha}\right) \mathrm{e}^{-b(1-\alpha) t}\right]+S(t)\left[\frac{a}{b+c}-\left(\frac{a}{b+c}-w_{\text {mat }}^{1-\alpha}\right) \mathrm{e}^{-(b+c)(1-\alpha)\left(t-t_{\text {mat }}\right)}\right]$

140 where $w_{0}$ is body weight at $t=0$ and $w_{\text {mat }}$ is body weight at $t=t_{\text {mat }}$ given by

$141 \quad w_{\mathrm{mat}}^{1-\alpha}=\frac{a}{b}-\left(\frac{a}{b}-w_{0}^{1-\alpha}\right) \mathrm{e}^{-b(1-\alpha) t_{\mathrm{mat}}}$.

142 The growth curve levels off at the asymptotic weight $w_{\infty}$,

$143 \quad w_{\infty}^{1-\alpha}=a /(b+c)$.

144 Total reproductive investment $R$ (including gonadic and behavioral costs) is obtained by

145 integrating the rate of energy conversion to reproduction from $t$ to $t+\Delta t$ :

$146 \quad R(t+\Delta t)=\int_{t}^{t+\Delta t} c w(\tau) \mathrm{d} \tau$

147 where $\Delta t$ describes the reproductive cycle over which the reproductive products are built

148 up, fertilized and cared until the offspring is autonomous. An analytical expression of $149 R(t+\Delta t)$ as a function of $w(t)$ and $w(t+\Delta t)$ can be obtained (Appendix A2). 150 Reproduction generally occurs at certain periods during lifespan. Fish for instance are 
151 often annual spawners (including North Sea plaice) and hence reproductive investment is 152 given over annual time steps $(\Delta t=1)$. Energy for reproduction is first stored in various

153 body tissues during the feeding period and then re-allocated to the gonad and released

154 during the spawning period. Since the currency of the model is energy, different energy 155 densities of different tissues have to be accounted for when fitting the model to real data.

156 Fitting procedure. The energy allocation model was fitted using a general-purpose 157 optimization procedure ( $\mathrm{R}$ 2.6., optim) by restricting all parameters to be positive using 158 box-constraints specification (Byrd, et al. 1995). Life history parameters $a, b, c$ and $159 t_{\text {mat }}$ were estimated by using this procedure to minimize the sum of squared residuals of 160 weight at age data versus predicted weight at age. Q-Q-plots indicated that the 161 distribution of residuals is close to normal. The algorithm was given a grid of possible 162 combinations of $a, b, c$ and $t_{\text {mat }}$ as starting values and the best solution was selected 163 based on the lowest AIC. A genetic algorithm (http://www.burns-stat.com/) yielded 164 similar results as those presented in this paper (not shown). The estimates of the time at 165 the onset of maturation $t_{\text {mat }}$ and the asymptotic weight $w_{\infty}^{1 / 4}=a /(b+c)$ were constrained 166 to a species-specific range (e.g. North Sea plaice $0.5 \mathrm{yr} \leq t_{\text {mat }} \leq 8.5 \mathrm{yr}, 400 \mathrm{~g} \leq w_{\text {mat }} \leq 4000 \mathrm{~g}$ ).

167 Confounding. Preliminary analyses of the plaice data set (see below) has shown that the 168 estimation of the 4 life history parameters $a, b, c$ and $t_{\text {mat }}$ (four-trait-estimation) yields 169 an unimodal distribution for energy acquisition $a$ but a bimodal distribution for 170 maintenance $b$ and reproductive investment $c$ (Figure 1). The mode in the distribution 171 of $b$ is likely an underestimation at 0 , which is related to an overestimation of $c$ 172 reflected in the $2^{\text {nd }}$ mode of its distribution. Selection of observations belonging to the $2^{\text {nd }}$ 
173 mode of the $b$ distribution thanks to a Gaussian mixture model (R 2.6., MClust, Fraley

174 and Raftery 2006) also removes the $2^{\text {nd }}$ mode in the $c$ distribution (dotted line, Figure 1 ).

175 To remove the confounding between $b$ and $c$ several options were considered: 1) use

176 only observations belonging to the $2^{\text {nd }} b$-mode - the correlation structure in these

177 observations was considered to be the most representative (Table 3) and was used for

178 simulations - or 2) assume parameter $b$ to be fixed at the population level (three-trait

179 estimation). The rationale for this choice is that maintenance costs are generally

180 acknowledged to be species- rather than individual-specific (Kooijman 2000) and our

181 main interest is in variation in reproductive investment. The population level value of $b$

182 was estimated by fitting a mean growth trajectory (Eq. 1) to the whole somatic weight-at-

183 age dataset. Confounding between $b$ and $c$ on this level was avoided by fitting

184 concomitantly reproductive investment $R(t+\Delta t)$ to an independent dataset of

185 reproductive investment-at-age (see application to real data). The partitioning of $b$ in the

186 sum $b+c$ could thereby be estimated accurately. The population mean growth trajectory

187 and reproductive investment were fitted simultaneously by minimizing the sum of

188 weighted squared residuals of somatic weight-at-age data and reproductive investment-at-

189 age data versus their predictions.

\section{$190 \quad$ PERFORMANCE ANALYSIS}

191 Performance. To test its performance, the method was applied to 2000 growth 192 trajectories simulated with known life history parameters. The life history parameters $a$, $193 b, c$ and $t_{\text {mat }}$ were drawn from a multivariate normal distribution with the co-variance 194 matrix taken from the results of the application to North Sea plaice data, after having 195 selected only observations belonging to the representative $b$-mode in the distribution of 
196 parameter estimates (see above, Table 3). To simulate weight-at-age data, $w(t+\Delta t)$ was

197 expressed as a function of $w(t)$ by using a function similar to Eq. (3) but in which

$198 w^{1-\alpha}(t)$ was replaced with $w^{1-\alpha}(t+\Delta t)$, and $w_{0}^{1-\alpha}$ (for $\left.t \leq t_{\mathrm{mat}}\right)$ and $w_{\mathrm{mat}}^{1-\alpha}$ (for $t>t_{\mathrm{mat}}$ )

199 with $w^{1-\alpha}(t)$. To evaluate the estimation bias on the population level and assess its

200 dependency on life-history strategy and environmental variability, the mean relative bias

201 over all life history parameters (the average absolute difference between estimated and

202 true values relative to true values) was used for each individual $i$ as a measure of

203 accuracy:

204

$e_{i}=\frac{1}{4}\left(\frac{\left|a_{\text {est }}-a_{\text {true }}\right|}{a_{\text {true }}}+\frac{\left|b_{\text {est }}-b_{\text {true }}\right|}{b_{\text {true }}}+\frac{\left|c_{\text {est }}-c_{\text {true }}\right|}{c_{\text {true }}}+\frac{\left|t_{\text {mat,est }}-t_{\text {mat, true }}\right|}{t_{\text {mat,true }}}\right)$

205 To test performance in the deterministic case, i.e. without environmental variability, the

206 bias $e_{i}$ was analyzed in dependence of a combination of two of the following factors: (i)

207 the relative reproductive investment $q=c /(b+c)$, (ii) the relative onset of maturation

$208 \tau=t_{\text {mat }}(b+c)$, (iii) the relative initial size $v_{0}=w_{0}(a /(b+c))^{-4}$, (iv) age $t$ and (v) the

209 number of observations in the mature stage $y_{\text {mat }}$. Variation in the three dimensionless

210 parameters $q, \tau$ and $v_{0}$ accounts for any variation in the parameters they are comprised

211 of, i.e. $a, b, c, t_{\text {mat }}$ and $w_{0}$, which allows investigating the whole parameter space at a

212 smaller cost.

213 Effects of temporal variability in environmental conditions. Individual growth

214 trajectories will be affected by environmental variability. To test whether the parameters

215 corresponding to energy acquisition $a$, maintenance $b$, reproductive investment $c$ and 
216 time at the onset of maturation $t_{\text {mat }}$ can be estimated reliably, annual stochasticity was

217 introduced in the individuals' life-history traits drawn from the multivariate normal 218 distribution (see deterministic case). As environmental variability is likely to be auto-

219 correlated, for simplicity a first order autoregressive process AR(1) was used to simulate 220 the lifespan series of the energy acquisition parameter $a$ (constrained to be positive):

$221 a_{t}=E(a)+\theta\left(a_{t-1}-E(a)\right)+\varepsilon_{t}$

$$
\varepsilon_{t} \sim N\left(0, \sigma_{a}^{2}\left(1-\theta^{2}\right)\right)
$$

222 where $E(a)$ denotes the expected value of $a, \theta$ is the autoregressive parameter and $\varepsilon_{t}$ is 223 a normally distributed noise term with mean 0 and variance $\sigma_{a}^{2}\left(1-\theta^{2}\right)$ where $\sigma_{a}^{2}$ is the 224 variance of $a_{t}$. The corresponding series of $b_{t}$ and $c_{t}$ were simulated by sampling $b_{t}$ 225 and $c_{t}$ from the normal distributions that yielded correlations with the autoregressive $a_{t}$ 226 series which were the same as those observed among the individual estimations. The 227 rationale here is that we assume that observed correlations between energy acquisition 228 and other life history traits across individuals are mainly due to plastic physiological 229 processes (versus genetic correlations) that therefore can also apply within individuals 230 through time in case of temporal variation in energy acquisition. More precisely, $b_{t}$ and $231 c_{t}$ were sampled from the normal distributions $N\left(\beta_{0}+\beta_{1} a_{t}, \sigma\right)$ that yielded linear 232 regressions of parameters $b$ and $c$ on $a$ that were consistent with observed means, 233 variances and correlations, that is with intercept $\beta_{0}$, slope $\beta_{1}$ and residual variance $\sigma^{2}$ 234 defined as:

235
$\beta_{0}=\bar{x}-\bar{a} \beta_{1}$
$\beta_{1}=\frac{\rho(a, x) \sigma_{x}}{\sigma_{a}}$
$\sigma^{2}=\sigma_{x}^{2}-\beta_{1}^{2} \sigma_{a}^{2}$ 
236 where $x$ stands for $b$ or $c$ and the means $\bar{x}$ and $\bar{a}$, variances $\sigma_{x}^{2}$ and $\sigma_{a}^{2}$, and 237 correlations $\rho(a, x)$ were taken from the empirical results of the application to North Sea 238 plaice $r(a, x)$ (see Table 3 ). Body weight was constrained to be monotonously increasing 239 while prioritizing reproduction over growth. Available surplus energy $a w^{3 / 4}-b w$ was 240 first allocated to reproduction and the remaining energy thereafter $a w^{3 / 4}-(b+c) w$ was 241 allocated to growth. If surplus energy happened to be negative $a w^{3 / 4}-b w<0$ acquisition $242 a$ and maintenance $b$ were resampled until obtaining a positive amount. If the remaining 243 energy was negative $a w^{3 / 4}-(b+c) w<0$, reproductive investment $c$ was adjusted such 244 that all available surplus energy was used for reproduction and none for somatic growth 245 by setting $c=a w^{-1 / 4}-b$. The initial conditions of the simulation were chosen such that 246 the realized $\theta_{a}$ of the initial at-series was within [0,1], and that the realized $C V$ 's in $a$, $247 \quad b$ and $c$ where within $[0,0.5]$. In addition to the relative reproductive investment $q$, 248 timing of onset of maturation $\tau$ and initial weight $v_{0}$, the effect of the expected value $249 E(x)$ of the parameters ( $x$ standing for $a, b$ or $c$ ), the realized coefficients of variation 250 of the parameters $C V_{x}$, the realized degree of auto-correlation $\theta_{x}$, and the realized 251 correlation $r_{\text {sim }}\left(x, x^{\prime}\right)$ between the simulated series of $a, b$ and $c$, the age $t$ and the 252 number of observations in the mature stage $y_{\text {mat }}$ on the mean of bias percentages (Eq. 5) 253 was analyzed by a linear model:

$254 e=\beta_{0}+\beta_{1} q+\beta_{2} \tau+\beta_{3} v_{0}+\beta_{4} t+\beta_{5} y_{\mathrm{mat}}+\beta_{6} C V_{x}+\beta_{7} \theta_{x}+\beta_{8} r\left(x, x^{\prime}\right)+\varepsilon$

255 where the $\beta$ 's are the statistical parameters and $\varepsilon$ is a normal error term (also in all 256 subsequent statistical models). In this case the true values of parameters $a, b$ and $c$ used 
257 for bias computation (see Eq. 5) were the geometric means of the respective realized time 258 series

259 Model uncertainty. The effect of uncertainty about the scaling exponent $\alpha$ of energy

260 acquisition rate with body weight was explored by fitting an energy allocation model to

261 the generated deterministic data set (i.e. without environmental noise) postulating a

262 scaling exponent lower $(\alpha=2 / 3)$ or higher $(\alpha=4 / 5)$ than the one used to generate the

263 data $(\alpha=3 / 4)$. A wrong assumption on $\alpha$ would lead to a different population level

264 estimate of the fixed $b$ and the effect of uncertainty about $\alpha$ in this approach was

265 explored along the same line as above.

\section{APPLICATION TO DATA}

267 Data. The method developed was applied to an empirical dataset of individual growth 268 trajectories back-calculated from otoliths of 1779 female North Sea plaice from cohorts 269 from the 1920s to the 1990s, aged at least 6 years (Rijnsdorp and Van Leeuwen 1992, 270 Rijnsdorp and Van Leeuwen 1996). This age threshold was chosen as these females then 271 have $90 \%$ probability of being sexually mature for at least one year (Grift, et al. 2003). 272 Because the otolith samples were length stratified, the observations of each length class 273 were weighted according to its relative frequency in the population to obtain population 274 level estimates.

275 Length-weight relationship. The back-calculated growth trajectories, which are in body 276 length units ( $l$ in $\mathrm{cm}$ ), were converted into body weight ( $w$ in $\mathrm{g}$ ). We used the 277 relationship between body weight $w$ and length $l$ of post spawning fish, estimated from 278 market sampling data by a linear model. The rationale was that spent fish have a low 279 condition, i.e. there are no energy reserves for reproduction in the post-spawning state: 
281 where $d$ is the day in the year accounting for the high condition early in the year before

282 spawning, the condition low after spawning and the building up of resources thereafter.

283 The body weight at $w_{0}=w(t=0)$ was assumed to be constant across individuals and

284 equal to $2.5 \mathrm{mg}$ corresponding to the weight of fish as large as the circumference of an 285 egg with a radius of $2 \mathrm{~mm}$ (Rijnsdorp 1991).

286 Maintenance. To avoid confounding between parameters, maintenance $b$ was assumed 287 to be fixed across individuals at its population level estimate (see section confounding 288 above). To obtain this estimate, the population mean growth trajectory and an 289 independent estimate of reproductive investment (see details below) were fitted 290 simultaneously by minimizing the sum of weighted squared residuals of somatic weight291 at-age data and reproductive investment-at-age data versus their predictions. The 292 population level estimates assuming the scaling exponent $\alpha=3 / 4$ were $a=4.84 . \mathrm{g}^{1 / 4} \cdot \mathrm{yr}^{-1}$, $293 b=0.47 \mathrm{yr}^{-1}, c=0.40 \mathrm{yr}^{-1}, t_{\text {mat }}=4.00 \mathrm{yr}$ (Figure 2). The population $b_{\alpha=3 / 4}=0.47 \mathrm{yr}^{-1}(\mathrm{see}$ 294 results) was used as a constant in the three-trait estimation.

295 Reproductive investment. Reproductive investment data included the cost of building 296 gonads as well as the cost of migration between the feeding and spawning grounds. 297 Reproductive investment $R_{\text {somatic }}$, expressed in units of energy-equivalent somatic weight, 298 was thus obtained as

$$
R_{\text {somatic }}=p_{\text {adult }}\left(g \kappa+M_{\text {resp }} / \delta\right)
$$


300 where $p_{\text {adult }}$ is the probability of being mature, $g$ is the gonad weight, $\kappa$ is the 301 conversion factor to account for different energy densities between gonad and soma, $M$

302 is the energy spent for migration and $\delta$ is the energy density of soma. Gonad weight $g$ 303 and the probability of being mature $p_{\text {adult }}$ were estimated as functions of size or age and 304 size, respectively, using linear models fitted to market samples of pre-spawning females:

$305 \log (g)=\beta_{0}+\beta_{1} \log (l)+\varepsilon$

306 Gonad weight was set to zero for females for which the probability of being mature $p_{\text {adult }}$

307 was less than 50\%, given age and size:

$308 \operatorname{logit}\left(p_{\text {adult }}\right)=\beta_{0}+\beta_{1} t+\beta_{2} l+\beta_{3} t \times l+\varepsilon$

309 The factor used to convert gonad weight $g$ to energy-equivalent somatic weight was $310 \kappa=1.75$, corresponding to the ratio between energy densities in pre-spawning gonad and 311 in post-spawning soma (Dawson and Grimm 1980). Migration cost was estimated 312 assuming a cruising speed $V$ of 1 body length per second (Videler and Nolet 1990). The 313 migration distance $D$ is positively related to body size (Rijnsdorp and Pastoors 1995) 314 with an average of about 140 nautical miles for a body length of $40 \mathrm{~cm}$ in plaice (Bolle, et 315 al. 2005). The energetic cost of swimming is then given by: $M_{\text {resp }}=\left(10^{0.3318} V(77.9 T+843.3) w^{3 / 4}\right) D / V$

317 where $M_{\text {resp }}$ is the respiration rate in $\mathrm{J}$ per month (Priede and Holliday 1980), $D / V$ is 318 the duration of active migration (in months) and $T$ is temperature in ${ }^{\circ} \mathrm{C}$, set to $10^{\circ} \mathrm{C}$. The 319 energy spent for respiration $M_{\text {resp }}$ was converted into energy-equivalent somatic weight 
320 assuming an energy density in post-spawning condition of $\delta=4.666 \mathrm{~kJ}^{-g^{-1}}$ (Dawson and

321 Grimm 1980).

322 The resulting size-dependent energy-based reproductive investment relative to the 323 somatic weight, i.e. the reproductive-somatic index RSI, increased with length $l$, and the 324 resulting gonadic investment relative to the reproductive investment, i.e. the gonado325 reproductive index GRI, was minimal for intermediate size classes (Figure 3). Using this 326 model, an average plaice of $40 \mathrm{~cm}$ length had a reproductive investment, expressed as a 327 percentage of the post-spawning body weight, of about $38.0 \%$, of which about $86 \%$ is 328 used for gonads and $14 \%$ for migration.

329 Validation. To validate the approach, the estimates of the time at the onset of maturation $330 t_{\text {mat }}$ were compared to independent estimates. Since $t_{\text {mat }}$ is estimated in continuous time 331 but reproduction occurs only at the start of the year, the age at first spawning $A_{\text {mat }}$ was 332 estimated by rounding up $t_{\text {mat }}$ to the next integer, assuming a minimal time interval of 4 333 months between the onset of maturation and the actual spawning season $\left(A_{\text {mat }}-t_{\text {mat }} \geq 1 / 3\right.$

334 year). These 4 months correspond to the minimal period of time during which gonads are 335 built up in typical annual spawners (Rijnsdorp 1990, Oskarsson, et al. 2002). From the 336 estimated $A_{\text {mat }}$, the probabilities of becoming mature at given ages and sizes were 337 estimated and compared to estimates obtained from independent population samples 338 (Grift, et al. 2003). Since the individuals' age at first spawning $A_{\text {mat }}$ was known, the 339 probability of becoming mature was estimated directly by logistic regression of the ratio 340 between the number of first time spawners and the number of juveniles plus first time 341 spawners (in population samples, first time and repeat spawners can usually not be 
342 distinguished and the fraction of first spawners has to be estimated separately). As in 343 Grift, et al. (2003), the probability of becoming mature was modeled as:

$344 \operatorname{logit}\left(p_{\text {mat }}\right)=\beta_{0}+\beta_{Y C} Y C+\beta_{t} t+\beta_{l} l+\beta_{Y C t} Y C \times t+\beta_{Y C l} Y C \times l+\beta_{t l} t \times l+\varepsilon$

345 i.e., the probability of becoming mature $p_{\text {mat }}$ depended on the individuals' year class $Y C$ 346 (cohort), age $t$ and length $l$. Year class was treated as a factor while age and length were 347 treated as continuous variables. The probability of becoming mature $p_{\text {mat }}$ is also referred 348 to as the probabilistic maturation reaction norm (PMRN, Heino, et al. 2002) and is 349 usually visualized using the 50\% probability isoline in the age-length plane (also referred 350 to as the PMRN midpoint or $\left.\mathrm{L}_{\mathrm{P} 50}\right)$. 


\section{PERFORMANCE ANALYSIS}

353 Parameter estimation in the deterministic case. When data are simulated

354 deterministically, i.e. without environmental noise, the bias in the life history parameter

355 estimation is negligible over the observed (estimated) range of values for both, the four-

356 trait and the three-trait estimation (Figure 4). The errors in the $b$-estimate are positively

357 correlated to errors in the estimates of $a$ and $t_{\text {mat }}$ and negatively correlated to errors in

358 the estimate of $c$ (Table 1), but this might not be very meaningful since the averages of

359 biases are about 0 . In the three-trait estimation, maintenance $b$ was assumed to be

360 constant to avoid confounding with reproductive investment $c$ (see below). For the four-

361 trait estimation, biases might arise if there are too few observations $y_{\text {mat }}$ of the mature

362 status, if the relative onset of maturation $\tau$ is early and if the relative reproductive

363 investment $q$ is small (Figure 4). The trends in the three-trait estimation are similar but

364 relative biases are lower and the relative influence of $q$ on the bias is much less

365 important (Figure 4).

366 Parameter estimation in the stochastic case. The suspected confounding between

367 maintenance $b$ and reproductive investment $c$ was confirmed by the results on simulated

368 data with environmental variability: 1) Although the co-variance structure used to

369 simulate data was taken from selected modes in the trait distribution estimated from real

370 data, the trait estimates obtained from these simulated data resulted in multimodal

371 distributions (Figure 5) very similar to those found in the estimates from real data (see 
372 Figure $1 \& 2)$. The estimation errors of $b$ and $c$ were negatively correlated $\left(r_{e}(b, c)=-\right.$

373 0.67, Table 1, Figure 5), whereas the bias in the sum of $b+c$ was much lower than in its

374 separate compounds $b$ and $c$ (18\% vs. -32 and $23 \%$ average deviation, Table 1, Figure

375 5). Hence, the sum $b+c$ is relatively well estimated but its partitioning between $b$ and $c$

376 is prone to error since an underestimation of maintenance $b$ is compensated by an

377 overestimation of reproductive investment $c$ and vice versa. This correlation between

378 estimation errors of $b$ and $c$ thus results in artifact modes in their trait distributions. If

$379 b+c$ is overestimated, acquisition $a$ has to be overestimated to fit a similar asymptotic

380 weight, therefore the high positive correlation between biases in $a$ and $b+c$

$381\left(r_{e}(a, b+c)=0.93\right.$, Table 1, Figure 5). Overestimation in $t_{\text {mat }}$ might compensate for

382 overestimation in $a$ or $b+c$ in the same way (not shown). The confounding could not be

383 removed by simply constraining the $b$-estimates above a certain positive threshold: the

384 parameter distribution turned out to be bimodal too, with the first mode around the

385 threshold instead of being around 0 (not shown). The unimodal distributions in the

386 deterministic case (not shown) indicate that confounding mainly arises due to the

387 interannual environmental stochasticity in the parameters along the growth trajectory.

388 Effects of environmental variability on parameter estimation. Environmental noise 389 increases the overall bias (Eq. 5). For four-trait estimation, bias most dramatically 390 increases with variation in the energy acquisition $C V_{a}$ as shown by the regression against 391 potentially explanatory variables (Eq. 8; Table 2). Furthermore, estimations are more 392 reliable, if relative reproductive investment $q$, the number of observations (age $t$ ), and 393 the correlation between $a$ and $b, r(a, b)$ are high but also if relative onset of maturation 
$394 \tau$ and the number of mature observations $y_{\text {mat }}$ are low (Table 2). In the three-trait 395 estimation, the signs of the effects of age $t$ and relative onset of maturation $\tau$ are 396 inversed, relative reproductive investment $q$ and the number of reproductive events do 397 not explain variation in bias but additional variation is explained by $C V_{c}$, the auto398 correlations $\theta_{a}$ and $\theta_{c}$ and the correlation $r(a, c)$ instead of $r(a, b)$.

399 Figure 6 shows the bias in the estimates of the life history parameters against the average 400 realized $C V$ 's. As expected, the variance and bias in the estimates typically increase with 401 the overall $C V$ (Figure 4) and the bias is on average higher in the four-trait estimation 402 than in the three-trait estimation. Generally, the variability in parameters results in an 403 underestimation of $a$ and $b$ and a slight overestimation in $t_{\text {mat }}$ relative to their mean 404 (Figure 6). Reproductive investment $c$ is generally overestimated relative to its 405 geometric mean in the four-trait estimation but slightly underestimated in the three-trait 406 estimation. Recall that the bias is defined relative to the realized geometric mean of the 407 parameter time series, and part of it may therefore not really represent estimation 408 inaccuracy since no real true value can be defined in this case (what is estimated does not 409 necessarily correspond to the geometric mean of the time series). Only the bias in $t_{\text {mat }}$ is 410 strictly defined here.

411 The age at onset of maturation $t_{\text {mat }}$ or age at first maturity $A_{\text {mat }}$ are generally 412 overestimated for the early maturing individuals (Table 4). This overestimation is smaller 413 in the three-trait estimation but on the other hand, many individuals are assigned to 414 mature at the earliest possible age in this approach. A very early maturation might be the 415 best solution in the energy allocation model fitting if no breakpoint can be detected in the 
416 growth curve. The confounding of parameters $a, b$ and $c$ does not seem to influence the

417 accuracy of $t_{\text {mat }}$-estimates significantly, since the similarity between confounded

418 estimates of $t_{\text {mat }}$ or $A_{\text {mat }}$ and estimates where the confounding has been removed is very

419 high (see below, Table 4).

420 Effect of model uncertainty on parameter estimation. Figure 7 shows the true against the

421 estimated values of the life history parameters in the deterministic case when the scaling 422 exponent $\alpha$ of energy acquisition rate with body weight was assumed to be lower ( $\alpha=$ $4232 / 3)$ or higher $(\alpha=4 / 5)$ in the model fitted to the data than in the one used to simulate 424 the data $(\alpha=3 / 4)$. For different scaling exponents, different population level estimates of 425 the parameters are obtained so that the value of fixed maintenance in the three-trait 426 estimation differs: $b_{\alpha=2 / 3}=0.33 \mathrm{yr}^{-1}, b_{\alpha=3 / 4}=0.47 \mathrm{yr}^{-1}, b_{\alpha=4 / 5}=0.88 \mathrm{yr}^{-1}$. Asymptotic body 427 weight $w_{\infty}^{1 / 4}=a /(b+c)$ is always estimated accurately (not shown). If $\alpha$ is assumed too 428 low $(\alpha=2 / 3)$, acquisition a and time at the onset of maturation $t_{\text {mat }}$ are generally 429 overestimated, whereas maintenance $b$ and reproductive investment $c$ are generally 430 underestimated and vice versa if $\alpha$ is assumed too high $(\alpha=4 / 5)$. The effect of an 431 erroneous assumption on the fixed value of maintenance $b$ in the three-trait estimation 432 was also evaluated. It had a negligible effect, resulting in a very small and constant bias 433 in parameters estimates for an assumption on b deviating by $10 \%$ from the true value (not 434 shown). APPLICATION to North Sea Plaice

436 The algorithm converged in $99 \%$ of the cases. The average estimates of life history 437 parameters, after removing the estimations corresponding to the artifact mode in the 
438 distribution of $b$ estimates, were $a=5.31 \mathrm{~g}^{1 / 4} \cdot \mathrm{yr}^{-1}, \quad b=0.57 \mathrm{yr}^{-1}, c=0.32 \mathrm{yr}^{-1}$ and $439 t_{\mathrm{mat}}=4.45 \mathrm{yr}$ (Table 3). Onset of maturation $t_{\mathrm{mat}}$ was negatively correlated with 440 acquisition $a, r\left(a, t_{\text {mat }}\right)=-0.22$, and reproductive investment $c, r\left(c, t_{\text {mat }}\right)=-0.63$, but 441 positively correlated with maintenance $b, r\left(b, t_{\text {mat }}\right)=0.30$ (Table 3 ). The correlation 442 between $a$ and $b+c$ was highly positive, $r(a, b+c)=0.93$. When using the three-trait 443 estimation procedure, i.e. assuming a maintenance fixed at its population level value $444 b=0.47$, the following average parameter estimates were obtained: $a=5.29 \mathrm{yr} \cdot \mathrm{g}^{-1-\alpha}$, $445 c=0.41 \mathrm{yr} \cdot \mathrm{g}^{-1}, t_{\mathrm{mat}}=3.53 \mathrm{yr}$ (Table 3). In this case, the correlation between $a$ and $t_{\text {mat }}$, $446 r\left(a, t_{\text {mat }}\right)=-0.68$, and between $a$ and $c, r(a, c)=0.91$, were stronger. The correlation 447 between $a$ and $c$ equals by definition the correlation between $a$ and $b+c$ under the 448 four-trait estimation (Table 3).

449 The four-trait and the three-trait estimation give roughly the same results for the timing of 450 maturation $t_{\text {mat }}$ or $A_{\text {mat }}$ (Table 4). The similarity of the $A_{\text {mat }}$ estimate between the two 451 approaches increases slightly, when only the observations belonging to the $2^{\text {nd }} b$-mode 452 are considered. The elimination of the confounding between maintenance $b$ and 453 reproductive investment $c$ by estimating only three traits or by selecting the $2^{\text {nd }} b$-mode 454 in the four-trait procedure does not affect the accuracy of the $t_{\text {mat }}$ estimate.

455 The probabilistic maturation reaction norms or PMRNs were derived only for cohorts $Y C$ 456 comprising at least 30 observations and showed a good match with those obtained by 457 Grift, et al. (2003) averaged over the same cohorts (Figure 8). For the maturation-relevant 458 ages, i.e. age 3 and 4, they are almost identical. The slope of the PMRN estimated here is 459 lower than the one in Grift, et al. (2003). 
461 Model assumptions. The method developed in this paper is the first to estimate

462 simultaneously the different life history parameters related to the energy allocation 463 schedule (energy acquisition, maintenance, onset of maturation and reproductive 464 investment) from individual growth trajectories. We restricted ourselves to a Von 465 Bertalanffy-like model, but, alternatively, structurally different energy allocation models, 466 such as net production or net assimilation models (Day and Taylor 1997, Kooijman 467 2000), could be used. The performance analysis shows that the method with a Von 468 Bertalanffy-like model can be expected to give accurate results as long as the scaling 469 exponents of the allometric relationships between the underlying energy allocation 470 processes (energy acquisition, maintenance, reproduction) and body weight applied in the 471 estimation are correct. Even if they are not, the results are still expected to be 472 qualitatively sound, and the resulting biases are predictable.

473 For the sake of simplicity, the scaling exponents of maintenance $\beta$ and reproductive 474 investment $\gamma$, here assumed to be 1 , were neither estimated nor tested for their effects on 475 estimation error, because a value different from 1 would require solving numerically the 476 differential equations describing energy allocation at each iteration. Applying equal 477 scaling exponents for energy acquisition and maintenance, i.e. $\alpha=\beta$, as suggested for 478 instance by Day and Taylor (1997) and Lester, et al. (2004), resulted in unrealistic 479 behavior of the energetic reproductive-somatic index RSI, suggesting that the scaling 480 exponent of maintenance needs to be higher than the exponent of energy acquisition. 
481 Based on theoretical (West, et al. 1997) and empirical case-specific evidence (Fonds, et 482 al. 1992), as well as on realistic asymptotic weight and RSI, we conclude that applying 483 scaling exponents following the inequalities $\alpha<\beta$ and $\alpha<\gamma$ are a good starting point

484 for the estimation of individual life history parameters.

485 Performance analysis. For practical applications, the method should be applied to data 486 on individuals for which two or more observations of the mature state are available. In 487 this case the estimation error is negligible in a deterministic setting over the range of 488 realistic (observed) parameter combinations. Environment variability in life history 489 parameters leads to a slight underestimation of the average parameters for energy 490 acquisition at and maintenance $b_{t}$ and an overestimation of reproductive investment $c_{t}$ 491 (not in the three-trait estimation) but the onset of maturation $t_{\text {mat }}$ is on average correctly 492 estimated. With increasing environmental noise the average biases increase (except for 493 the maintenance $b$ ) and estimation precision decreases (Figure 4). Variability in $a_{t}$ has 494 the largest impact on bias and the relative reproductive investment $q$ might have to stay 495 above a certain level to minimize the bias (Table 2). The negative effect on the bias of 496 age is balanced by a positive effect of relative onset of maturation $\tau$ and of the number 497 of adult observations $y_{\text {mat }}$ and the interpretation of the deterministic case, where $y_{\text {mat }}$ had 498 a negative effect on the bias, therefore not necessarily falsified. However, these biases 499 should be interpreted with caution because they were computed relative to the geometric 500 mean of the simulated parameter time series, which does not correspond to a 'true' value 501 as in the deterministic case. In other terms, there is no natural 'true' value to be compared 502 with estimates in the stochastic case, except for $t_{\text {mat }}$. 
503 Life-history correlation. (Co-)variation in (between) life history parameters at the 504 phenotypic level, i.e. as observed across individuals, results from a genetic and an 505 environmental (plastic) source of (co-)variation (Lynch and Walsh 1998). From life 506 history theory (Roff 1992, Stearns 1992) we expect that 1i) juvenile growth rate $507 \partial w_{\text {juv }} / \partial t$ and age at maturation $t_{\text {mat }}$ are negatively correlated $\rho\left(\partial w_{\text {juv }} / \partial t, t_{\text {mat }}\right) \leq 0-$ the 508 higher the juvenile growth rate is, the earlier the individual will hit a presumably fixed 509 genetically determined PMRN and mature - and 1ii) size-specific reproductive 510 investment RSI and age at maturation $t_{\text {mat }}$ are negatively correlated $\rho\left(\mathrm{RSI}, t_{\text {mat }}\right) \leq 0$. 511 From the assumptions of our bioenergetic model it is given that $2 \mathrm{i}$ ) juvenile growth rate $512 \partial w_{\text {juv }} / \partial t$ increases with size-specific energy acquisition rate $a$, resulting in a positive 513 correlation $\left.\rho\left(\partial w_{\text {juv }} / \partial t, a\right) \geq 0 ; 2 \mathrm{ii}\right)$ juvenile growth rate $\partial w_{\text {juv }} / \partial t$ decreases with size514 specific maintenance rate $b$, resulting in a negative correlation $\rho\left(\partial w_{\text {juv }} / \partial t, b\right) \leq 0$; and 515 2iii) size-specific reproductive investment RSI increases with size-specific reproductive 516 investment rate $c$, resulting in a positive correlation $\rho(\mathrm{RSI}, c) \geq 0$. Life history theory 517 and our model assumptions together thus lead to the following expectations: $3 \mathrm{i}$ ) size518 specific energy allocation rate $a$ is negatively correlated with age at maturation $t_{\text {mat }}$, $\left.519 \rho\left(a, t_{\mathrm{mat}}\right) \leq 0 ; 3 \mathrm{ii}\right)$ size-specific maintenance rate $b$ is positively correlated with age at 520 maturation $t_{\text {mat }}, \rho\left(b, t_{\text {mat }}\right) \geq 0$; and 3iii) size-specific reproductive investment rate $c$ is 521 negatively correlated with age at maturation $t_{\text {mat }}, \rho\left(c, t_{\text {mat }}\right) \leq 0$. The correlations between $522 a, b$ and $c$ cannot be easily interpreted in terms of life history theory but can be in the 523 light of our model: since the asymptotic size $w_{\infty}^{1 / 4}=a /(b+c)$ is roughly constant within 524 a species, increases in size-specific energy acquisition $a$ or in speed of growth $(b+c)$ 
525 are reciprocally compensated to stabilize $w_{\infty}$. The construction of the model therefore

526 imposes $\rho(b, c) \leq 0$ and $\rho(a, b+c) \geq 0$, the only degrees of freedom being $\rho(a, c)$ and $527 \rho(a, b)$.

528 In terms of environmental variation, energy acquisition $a$ might be externally influenced 529 by variable food availability, maintenance $b$, interpreted here as the resting metabolic 530 rate (i.e. the increase in maintenance due to higher consumption is accounted for by $a$ ), 531 might be externally influenced by variability in temperature only and reproductive 532 investment $c$ might vary with the annually stored energy resources. From the 533 environmental co-variation, the correlations $\rho(a, c)$ and $\rho(a, b)$ might be expected 534 across individuals and within the lifespan of an individual: the positive effect of 535 temperature on both food availability due to increased productivity of the system, and 536 hence $a$, and metabolic rates, hence $b$, may lead to a positive correlation $\rho(a, b) \geq 0$; 537 the energy resources available for reproductive investment (gonadic tissue, spawning 538 migration) is determined by the energy which is physiologically made available and 539 hence likely mainly by $a$, causing a positive correlation $\rho(a, c) \geq 0$ on the phenotypic 540 level according to the rule "the more resources are available, the more can be spent".

541 The signs of the correlations between life history parameters obtained for plaice (Table 3) 542 matched the previous theoretical expectations. Most importantly, we find $r\left(a, t_{\text {mat }}\right) \leq 0$, $543 r\left(b, t_{\text {mat }}\right) \geq 0$ and $r\left(c, t_{\text {mat }}\right) \leq 0$. These correlations also might be to some degree due to 544 the correlation between estimation errors (Table 1) but not entirely, since the correlations 545 between the traits are higher than between the errors (and the absolute traits are larger 546 than the errors). The correlations $r(b, c)$ and $r(a, b+c)$ are indeed found to be due to the 
547 correlations between estimation errors (Table 1) and thereby contribute, by construction

548 of the model, to stabilize the asymptotic weight $w_{\infty}$ (see above). The $r(a, b)$ might also

549 be partly due to the error correlation. However, $r(a, c)$ is not, since the errors in $a$ and $c$

550 are negatively correlated, whereas the found $r(a, c)$ is about 0 . This indicates that the

551 true $r(a, c)$ might in fact be positive. In the three-trait estimation, $r(a, c)=0.91$ is indeed

552 highly positive, suggesting that the $r(a, c)$ found in the four-trait estimation might be due

553 to the confounding with maintenance rate $b$. By assuming $a$ constant $b$ in the three-trait

554 estimation, the co-variances between the three traits $a, c$ and $t_{\text {mat }}$ are inflated. The 555 correlation $r(a, c)$ in the three-trait estimation becomes equal to the correlation $556 r(a, b+c)$ in the four-trait estimation, due to the classical relationship of covariances $557 \operatorname{cov}(a, b+c)=\operatorname{cov}(a, b)+\operatorname{cov}(a, c)$. In the three-trait estimation $\operatorname{cov}(a, c)$ is inflated by 558 artificially fixing $b$ and thereby forcing the covariance $\operatorname{cov}(a, b)=0$ to nullity so that $559 \operatorname{cov}(a, b+c)=\operatorname{cov}(a, c)$.

560 Application to real data. The method validation was based on the comparison between 561 estimates of the timing of the onset of maturation $t_{\text {mat }}$ obtained from back-calculated 562 growth trajectories and independent estimates obtained from biological samples from the 563 spawning population. Both estimation procedures are subject to error but similar patterns 564 should nevertheless indicate the likelihood of both. For the ages at which maturation 565 mainly occurs (around age 4), the PMRN based on our estimates is very similar to the 566 PMRNs based on biological samples from the population (Grift, et al. 2003). The 567 relatively higher and lower maturation probability for younger and older ages 568 respectively is likely due to extrapolation to ages at which only few fish become mature 
569 and the estimation becomes less reliable. If the interval between the start of energy

570 allocation to reproduction $t_{\text {mat }}$ and the subsequent age at first spawning $A_{\text {mat }}$ was

571 assumed to be less or more than 4 months, the resulting reaction norm would be lower or

572 higher respectively in the age-size plane. However, for plaice 4 months correspond to the

573 time interval between the onset of vitellogenesis (August, September) and the midpoint

574 of the spawning season (Rijnsdorp 1990, Oskarsson, et al. 2002). The good

575 correspondence between the two estimation methods of the PMRN suggests that

576 environmental variability is unlikely to have been so high as to result in biases as high as

577 in the simulation analysis (see biases of $t_{\mathrm{mat}}$ in Figure 4).

578 Reproductive investment. Reproductive investment was modeled including a size579 dependent gonadic investment and a size-dependent cost of migration. The modeled 580 energetic reproductive-somatic index RSI (energy-based reproductive investment relative 581 to somatic weight) is increasing with somatic weight as is the modeled gonado582 reproductive index GRI (gonadic relative to reproductive investment) and consequently 583 the resulting gonado-somatic index GSI (gonadic weight relative to somatic weight). This 584 is in line with the expectation since data show that GSI increases with size (Rijnsdorp 585 1991). In contrast, the modeled migration cost relative to reproductive investment (1586 GSI) decreases with size. Since migration distance increases with fish size (Rijnsdorp and 587 Pastoors 1995, Bolle, et al. 2005), the advantage of feeding offshore must be relatively 588 more important than the migration cost.

589 Possible extensions. The method proposed here can be applied to a variety of organisms 590 in which the annual pattern in somatic growth is reflected in hard structures: scales or 591 otoliths in fish (Rijnsdorp, et al. 1990, Panfili and Tomas 2001, Colloca, et al. 2003), 
592 shells in bivalves (Witbaard, et al. 1997, Witbaard, et al. 1999), endoskeleton in 593 echinoderms (Pearse and Pearse 1975, Ebert 1986, Gage 1992), teeth in mammals (Laws 594 1952, Godfrey, et al. 2001, Smith 2004) or skeleton in amphibians (Misawa and Matsui 595 1999, Kumbar and Pancharatna 2001) and reptiles (Zug, et al. 2002, Snover and Hohn 596 2004). If a back-calculation method from the hard structures can be validated, the 597 analysis of individual growth trajectories with the method developed in this paper offers 598 the opportunity to study a variety of life history trade-offs without the need to follow 599 individuals throughout their lifetime using experiments in controlled conditions or 600 methods such as mark-recapture. The method holds for any other frequency of age and 601 size observations and for any other frequency of spawning than the here illustrated annual 602 observations and annual spawning intervals. Under the assumption that energy is 603 allocated to reproduction continuously between spawning events by storing energy 604 reserves which are then made available later for spawning, the method even applies if 605 spawning intervals are irregular.

606 Adaptation. Our method could be particularly useful to study changes in life history 607 parameters over time or differences among populations. Concerns had been raised that 608 life history traits of exploited species, may evolve in response to harvesting (Rijnsdorp 609 1993, Stokes, et al. 1993, Heino 1998, Law 2000). Studies on life history evolution in the 610 wild have largely focused on changes in the onset of maturation, although evolutionary 611 changes were also suggested in growth rate and reproductive investment (see review in 612 Jørgensen, et al. 2007). The analysis of harvesting-induced evolution in the wild has 613 proved to be difficult (Rijnsdorp 1993, Law 2000, Sinclair, et al. 2002, Conover, et al. 614 2005). One reason is that growth, maturation and reproductive investment are intricately 
615 linked in the energy allocation schedule, another that disentangling phenotypic plasticity

616 from genetic effects in the observed phenotypic response is not evident

617 Disentangling plasticity. By estimating the co-variance structure between the life history

618 parameters, our method may prove useful to disentangle phenotypic plasticity from

619 genetic change. Assuming that environmental variability mostly affects the primary

620 energy flow of energy acquisition and that the subsequent energy allocations

621 (maintenance, reproductive investment) are partly determined by this primary energy

622 flow, plastic variation in the other traits due to this process could be accounted for by

623 expressing them conditional on energy acquisition. It is for instance likely that

624 reproductive investment may be affected by feeding conditions during the previous

625 growing season (Rijnsdorp 1990, Stearns 1992, Kjesbu, et al. 1998, Marshall, et al.

626 1999). Studies in other taxa than fish (e.g. Ernande, et al. 2004) have shown that the

627 energy allocation strategy between maintenance, growth, and reproductive investment

628 may vary according to food availability. Expressing reproductive investment conditional

629 on energy acquisition would therefore represent a reaction norm for reproductive

630 investment (Rijnsdorp, et al. 2005). Changes in this reaction norm would then reveal

631 genetic change under the assumption that most environmental influence on reproductive

632 investment is accounted for via variation in energy acquisition. It has also been shown

633 here that the PMRN can be estimated directly from the back-calculated ages and sizes

634 and the obtained estimate for the age at first maturity, whereas in other data sources the

635 individual first maturity is typically not known (see Barot, et al. 2004). By disentangling

636 the plasticity in maturation caused by variation in growth and removing the effect of

637 survival on observed maturation events, the PMRN can also be used to assess genetic 
638 changes under the assumption that most environmental influence on maturation is 639 accounted for via growth variation.

640 Different approaches. In an earlier study, Rijnsdorp and Storbeck (1995) estimated the

641 timing of the onset of maturation in plaice by piecewise linear regression of growth

642 increments on body weight to locate the discontinuity in growth rates expected at

643 maturation. This method might be accurate only for particular combinations of the energy

644 allocation scaling exponents that lead to a linear relationship between growth increments

645 and body weight (not shown). Baulier and Heino (2008) applied an improved version of

646 this method to Norwegian spring spawning herring and obtained relatively accurate

647 estimates ( \pm 1 year) of the timing of the onset of maturation. However, this method does

648 not provide estimates of the other life history parameters and it is unlikely that the 649 particular combination of energy allocation scaling exponents leading to the 650 discontinuous linear relationship between growth increments and body weight can be 651 expected to apply in the general case.

652 The three-trait estimation procedure in the method presented in this paper removes the 653 confounding between parameters by fixing maintenance to its population level average. 654 However, in reality maintenance may be variable since it is affected for instance by 655 temperature and, in addition, assuming a fixed value inflates co-variances between other 656 parameters. A more elegant way to circumvent this problem may be to use generalized 657 linear mixed modeling to estimate the four parameters. Under this approach, the 658 parameters, shown here to be approximately normally distributed after removal of the 659 confounded estimates (Fig.1), follow a multivariate normal distribution and estimation 
660 can thus only lead to unimodal distributions, therefore potentially reducing the

661 confounding between parameters (Brunel, et al. submitted).

662 The four-trait method presented in this paper is not practical, since the first mode in the

663 distribution of $b$ estimates would always have to be removed $a$ posteriori. The three-trait

664 estimation gives more stable results (Figures 4, 5 and 6) but a correction for changing

665 temperatures would be needed (see below) and due to the inflation of co-variances,

666 results should be considered on a relative scale. If the main interest is on the onset of

667 maturation $t_{\text {mat }}$, then both four-trait and three-trait estimation work similarly well, since

668 the bias in $t_{\text {mat }}$ is unlikely due to confounding in the parameters $a, b$ and $c$ (Table 4,

669 Figure 4).

670 Maintenance-Temperature. The estimated energy allocation parameters here represent 671 average values for the study period. However, assuming a constant maintenance (three-

672 trait estimation) may be incorrect as yearly averaged surface temperatures in the North

673 Sea (Van Aken 2008) suggest that temperature increased from $9.91^{\circ} \mathrm{C}$ in 1950 to $11.01^{\circ} \mathrm{C}$

674 in $2005(\mathrm{p}<0.001)$. In the interpretation used here, the size-specific maintenance is

675 influenced only by temperature. The Arrhenius description based on the Van't Hoff

676 equation used in dynamic energy budget modeling (Van der Veer, et al. 2001) to describe

677 the effect of temperature on physiological rates would predict that an increase from $10^{\circ} \mathrm{C}$

678 to $11^{\circ} \mathrm{C}$ would correspond to an increase in the maintenance rate of about $9 \%$ (not

679 shown). If a similar trend occurred in the bottom temperatures, we might expect a change

680 in the average maintenance cost over the study period of about $9 \%$. In the three-trait

681 estimation, the trend in temperature could therefore be accounted for by estimating a 682 separate average $b$ for each cohort. As this paper explores average general patterns, we 
683 ignored here the effect of temperature on maintenance by assuming homogeneous 684 temperatures in the demersal zone.

685 Conclusion. This paper is the first one to present a method to estimate the energy 686 allocation parameters for energy acquisition, maintenance, reproductive investment and 687 onset of maturation of organisms from individual growth trajectories. Performance 688 analysis and the application to real data showed that the method can be successfully 689 applied, at least on a qualitative level, to estimate the relative differences in energy 690 allocation parameters between individuals and to estimate their co-variance structure. 691 Future studies will apply the concept to back-calculated growth curves from otoliths of 692 North Sea sole and plaice and scales of Norwegian spring spawning herring, focusing on 693 the comparison between species and life-history adaptation over the last century. 
695 This research has been supported by the European research training network FishACE 696 funded through Marie Curie (contract MRTN-CT-2204-005578). We thank Ulf 697 Dieckmann and Mikko Heino for conceptual advice, Christian Jørgenson for support in 698 considerations on metabolism, Patrick Burns (http://www.burns-stat.com/) for 699 consulting in algorithm programming, Henk Van der Veer for clarifying the dependence 700 of maintenance on temperature in North Sea plaice, and all members of the FishACE and 701 FinE networks for useful discussions. 
703 Banavar, J. R., Damuth, J., Maritan, A. and Rinaldo, A. 2002. Supply-demand balance and metabolic scaling. - Proceedings of the National Academy of Sciences 99: 10506-10509.

Barot, S., Heino, M., O'Brien, L. and Dieckmann, U. 2004. Estimating reaction norms for age and size at maturation when age at first reproduction is unknown. Evolutionary Ecology Research 6: 659-678.

Baulier, L. and Heino, M. 2008. Norwegian spring-spawning herring as the test case of piecewise linear regression method for detecting maturation from growth patterns. - Journal of Fish Biology 73: 2452-2467.

712 Blueweiss, L., Fox, H., Kudzma, V., Nakashima, D., Peters, R. and Sams, S. 1978. Relationships between body size and some life-history parameters. - Oecologia 37: 257-272.

Bolle, L. J., Hunter, E., Rijnsdorp, A. D., Pastoors, M. A., Metcalfe, J. D. and Reynolds, J. D. 2005. Do tagging experiments tell the truth? Using electronic tags to evaluate conventional tagging data. - ICES Journal of Marine Science 62: 236-

Brunel, T., Ernande, B., Mollet, F. M. and Rijnsdorp, A. D. 2009. Estimating age at 246.

Brown, J. H., Gillooly, J. F., Allen, A. P., Savage, V. M. and West, G. B. 2004. Toward a metabolic theory of ecology. - Ecology 85: 1771-1789. maturation and energy-based life-history traits from individual growth trajectories with nonlinear mixed-effects models. - Journal of Animal Ecology submitted. 
724 Byrd, R. H., Lu, P., Nocedal, J. and Zhu, C. 1995. A limited memory algorithm for bound constrained optimization. - SIAM J. Scientific Computing 16: 1190-1208.

726 Charnov, E. L., Turner, T. F. and Winemiller, K. O. 2001. Reproductive constraints and the evolution of life histories with indeterminate growth. - Proceedings of the National Academy of Sciences of the United States of America 98: 9460-9464.

Clarke, A. 2004. Is there a universal temperature dependence of metabolism? Functional Ecology 18: 252-256.

731 Colloca, F., Cardinale, M., Marcello, A. and Ardizzone, G. D. 2003. Tracing the life history of red gurnard (Aspitrigla cuculus) using validated otolith annual rings. -

734 Conover, D. O., Arnott, S. A., Walsh, M. R. and Munch, S. B. 2005. Darwinian fishery 735 science: lessons from the Atlantic silverside (Menidia menidia). - Canadian

737 Darveau, C. A., Suarez, R. K., Andrews, R. D. and Hochachka, P. W. 2002. Allometric cascade as a unifying principle of body mass effects on metabolism. - Nature 417: 166-170.

740 Dawson, A. S. and Grimm, A. S. 1980. Quantitative changes in the protein, lipid and energy content of the carcass, ovaries and liver of adult female plaice, Pleuronectes platessa L. - Journal of Fish Biology 16: 493-504.

743 Day, T. and Taylor, P. D. 1997. Von Bertalanffy's growth equation should not be used to model age and size at maturity. - American Naturalist 149: 381-393.

745 Ebert, T. A. 1986. A new theory to explain the origin of growth lines in sea urchin spines. - Marine Ecology-Progress Series 34: 197-199. 
747 Engelhard, G. H., Dieckmann, U. and Gødo, O. R. 2003. Age at maturation predicted from routine scale measurements in Norwegian spring-spawning herring (Clupea harengus) using discriminant and neural network analyses. - ICES Journal of Marine Science 60: 304-313.

751 Ernande, B., Boudry, P., Clobert, J. and Haure, J. 2004. Plasticity in resource allocation based life history traits in the Pacific oyster, Crassostrea gigas. I. Spatial variation in food abundance. - Journal of Evolutionary Biology 17: 342-356.

754 Fonds, M., Cronie, R., Vethaak, A. D. and Van der Puyl, P. 1992. Metabolism, food consumption and growth of plaice (Pleuronectes platessa) and flounder (Platichthys flesus) in relation to fish size and temperature. - Netherlands Journal

758 Fraley, C. and Raftery, A. E. 2006. MCLUST Version 3 for R: Normal mixture modeling and model-based clustering. - Department of Statistics, University of Washington Technical Report No. 504.

761 Francis, R. and Horn, P. L. 1997. Transition zone in otoliths of orange roughy (Hoplostethus atlanticus) and its relationship to the onset of maturity. - Marine Biology 129: 681-687.

764 Gage, J. D. 1992. Natural growth bands and growth variability in the sea urchin Echinus Esculentus - results from tetracycline tagging. - Marine Biology 114: 607-616.

766 Gillooly, J. F., Brown, J. H., West, G. B., Savage, V. M. and Charnov, E. L. 2001. Effects of size and temperature on metabolic rate. - Science 293: 2248-2251. 
Godfrey, L. R., Samonds, K. E., Jungers, W. L. and Sutherland, M. R. 2001. Teeth, brains, and primate life histories. - American Journal of Physical Anthropology 114: 192-214.

Grift, R. E., Rijnsdorp, A. D., Barot, S., Heino, M. and Dieckmann, U. 2003. Fisheriesinduced trends in reaction norms for maturation in North Sea plaice. - Marine Ecology Progress Series 257: 247-257.

Heino, M. 1998. Management of evolving fish stocks. - Canadian Journal of Fisheries and Aquatic Sciences 55: 1971-1982.

Heino, M. and Kaitala, V. 1999. Evolution of resource allocation between growth and reproduction in animals with indeterminate growth. - Journal of Evolutionary Biology 12: 423-429.

Heino, M., Dieckmann, U. and Godo, O. R. 2002. Measuring probabilistic reaction norms for age and size at maturation. - Evolution 56: 669-678.

Jørgensen, C., Enberg, K., Dunlop, E. S., Arlinghaus, R., Boukal, D. S., Brander, K., Ernande, B., Gardmark, A., Johnston, F., Matsumura, S., Pardoe, H., Raab, K., Silva, A., Vainikka, A., Dieckmann, U., Heino, M. and Rijnsdorp, A. D. 2007. Ecology - Managing evolving fish stocks. - Science 318: 1247-1248.

Kjesbu, O. S., Witthames, P. R., Solemdal, P. and Walker, M. G. 1998. Temporal variations in the fecundity of Arcto-Norwegian cod (Gadus morhua) in response to natural changes in food and temperature. - Journal of Sea Research 40: 303321. 
Kooijman, S. A. L. M. 1986. Population dynamics on basis of energy budgets. - In: Metz, J. A. J. and Dieckmann, O. (eds.), The dynamics of physiologically structured populations. Springer-Verlag, pp. 266-297.

792 Kooijman, S. A. L. M. 2000. Dynamic energy and mass budgets in biological systems. Cambridge University Press.

794 Kozlowski, J. 1996. Optimal allocation of resources explains interspecific life history patterns in animals with indeterminate growth. - Proceedings of The Royal Society of London Series B-Biological Sciences 263: 559-566.

797 Kozlowski, J. and Konarzewski, M. 2004. Is West, Brown and Enquist's model of allometric scaling mathematically correct and biologically relevant? - Functional Ecology 18: 283-289.

800 Kumbar, S. M. and Pancharatna, K. 2001. Determination of age, longevity and age at 801 reproduction of the frog Microhyla ornata by skeletochronology. - Journal of

803 Law, R. 2000. Fishing, selection, and phenotypic evolution. - ICES Journal of Marine 804 Science 57: 659-668.

805 Laws, R. M. 1952. A new method of age determination for mammals. - Nature 169: 972806 973.

807 Lester, N. P., Shuter, B. J. and Abrams, P. A. 2004. Interpreting the von Bertalanffy 808 model of somatic growth in fishes: the cost of reproduction. - Proceedings of the

810 Lynch, M. and Walsh, B. 1998. Genetics and analysis of quantitative traits. - Sunderland: Sinauer Associates Inc. 
812 Marshall, C. T., Yaragina, N. A., Lambert, Y. and Kjesbu, O. S. 1999. Total lipid energy as a proxy for total egg production by fish stocks. - Nature 402: 288-290.

814 Misawa, Y. and Matsui, M. 1999. Age determination by skeletochronology of the Japanese salamander Hynobius kimurae (Amphibia, Urodela). - Zoological Science 16: 845-851.

817 Oskarsson, G. J., Kjesbu, O. S. and Slotte, A. 2002. Predictions of realised fecundity and spawning time in Norwegian spring-spawning herring (Clupea harengus). Journal of Sea Research 48: 59-79.

Panfili, J. and Tomas, J. 2001. Validation of age estimation and back-calculation of fish length based on otolith microstructures in tilapias (Pisces, Cichlidae). - Fishery Bulletin 99: 139-150.

Pearse, J. S. and Pearse, V. B. 1975. Growth zones in echinoid skeleton. - American Zoologist 15: 731-753.

Priede, I. G. and Holliday, F. G. T. 1980. The use of a new tilting tunnel respirometer to 826 investigate some aspects of metabolism and swimming activity of the plaice

828 Rijnsdorp, A. D. 1990. The mechanism of energy allocation over reproduction and somatic growth in female North-Sea plaice, Pleuronectes platessa L. -

831 Rijnsdorp, A. D. 1991. Changes in fecundity of female North-Sea plaice (Pleuronectes platessa L) between 3 periods since 1900. - ICES Journal of Marine Science 48: 253-280. 
834 Rijnsdorp, A. D. 1993. Fisheries as a large-scale experiment on life history evolution disentangling phenotypic and genetic effects in changes in maturation and reproduction of North Sea plaice, Pleuronectes platessa L. - Oecologia 96: 391401.

Rijnsdorp, A. D. and Van Leeuwen, P. I. 1992. Density-dependent and independent changes in somatic growth of female North Sea plaice Pleuronectes platessa between 1930 and 1985 as revealed by back-calculation of otoliths. - Marine Ecology-Progress Series 88: 19-32.

842 Rijnsdorp, A. D. and Pastoors, M. A. 1995. Modeling the spatial dynamics and fisheries of North Sea plaice (Pleuronectes Platessa L) based on tagging data. - ICES Journal of Marine Science 52: 963-980.

Rijnsdorp, A. D. and Storbeck, F. 1995. Determining the onset of sexual maturity from otoliths of individual female North Sea plaice, Pleuronectes platessa L. - In:

849 Rijnsdorp, A. D. and Van Leeuwen, P. I. 1996. Changes in growth of North Sea plaice Secor, D. H., Dean, J. M. and S., C. (eds.), Recent Developments in Fish Otolith

852 Rijnsdorp, A. D., Van Leeuwen, P. I. and Visser, T. A. M. 1990. On the validity and precision of back-calculation of growth from otoliths of the plaice, Pleuronectes platessa L. - Fisheries Research 9: 97-117. 
855 Rijnsdorp, A. D., Grift, R. E. and Kraak, S. B. M. 2005. Fisheries-induced adaptive change in reproductive investment in North Sea plaice (Pleuronectes platessa)? Canadian Journal of Fisheries and Aquatic Sciences 62: 833-843.

Roff, D. A. 1991. The evolution of life-history variation in fishes, with particular reference to flatfishes. - Netherlands Journal of Sea Research 27: 197-207.

861

Roff, D. A. 1992. The evolution of life histories. - Chapman \& Hall.

Runnström, S. 1936. A study on the life history and migrations of the Norwegian springspawning herring based on the analysis of the winter rings and summer zones of the scale. - Fisk Dir. Skr. Ser. Havunders. 5: 1-103.

Savage, V. M., Gillooly, J. F., Woodruff, W. H., West, G. B., Allen, A. P., Enquist, B. J. and Brown, J. H. 2004. The predominance of quarter-power scaling in biology. Functional Ecology 18: 257-282.

Sinclair, A. F., Swain, D. P. and Hanson, J. M. 2002. Measuring changes in the direction and magnitude of size-selective mortality in a commercial fish population. Canadian Journal of Fisheries and Aquatic Sciences 59: 361-371.

Smith, S. L. 2004. Skeletal age, dental age, and the maturation of KNM-WT 15000. American Journal of Physical Anthropology 125: 105-120.

Snover, M. L. and Hohn, A. A. 2004. Validation and interpretation of annual skeletal marks in loggerhead (Caretta caretta) and Kemp's ridley (Lepidochelys kempii) sea turtles. - Fishery Bulletin 102: 682-692.

Stearns, S. C. 1992. The evolution of life histories. - Oxford University Press.

Stevenson, R. D. and Woods Jr., W. A. 2006. Condition indices for conservation: New uses for evolving tools. - Integrative and Comparative Biology 46: 1169-1190. 
Stokes, T. K., McGlade, J. M. and Law, R. 1993. The exploitation of evolving resources. - Springer-Verlag.

Van Aken, H. M. 2008. Variability of the water temperature in the western Wadden Sea on tidal to centennial time scales. - Journal of Sea Research 60: 227-234.

Van der Veer, H. W., Kooijman, S. A. L. M. and Van der Meer, J. 2001. Intra- and interspecies comparison of energy flow in North Atlantic flatfish species by means of dynamic energy budgets. - Journal of Sea Research 45: 303-320.

Videler, J. J. and Nolet, B. A. 1990. Costs of swimming measured at optimum speed scale effects, differences between swimming styles, taxonomic groups and submerged and surface swimming. - Comparative Biochemistry and Physiology a-Physiology 97: 91-99.

Von Bertalanffy, L. and Pirozynski, W. J. 1952. Tissue respiration, growth, and basal metabolism. - Biological Bulletin 105: 240-256.

Ware, D. M. 1982. Power and evolutionary fitness of teleosts. - Canadian Journal of Fisheries and Aquatic Sciences 39: 3-13.

West, G. B., Brown, J. H. and Enquist, B. J. 1997. A general model for the origin of allometric scaling laws in biology. - Science 276: 122-126.

West, G. B., Brown, J. H. and Enquist, B. J. 1999. The fourth dimension of life: Fractal geometry and allometric scaling of organisms. - Science 284: 1677-1679.

West, G. B., Brown, J. H. and Enquist, B. J. 2001. A general model for ontogenetic growth. - Nature 413: 628-631. 
899 Witbaard, R., Franken, R. and Visser, B. 1997. Growth of juvenile Arctica islandica 900 under experimental conditions. - Helgolander Meeresuntersuchungen 51: 417901 431.

902 Witbaard, R., Duineveld, G. C. A. and de Wilde, P. 1999. Geographical differences in 903 growth rates of Arctica islandica (Mollusca : Bivalvia) from the North Sea and 904 adjacent waters. - Journal of the Marine Biological Association of the United 905 Kingdom 79: 907-915.

906 Zug, G. R., Balazs, G. H., Wetherall, J. A., Parker, D. M. and Murakawa, S. K. K. 2002. 907 Age and growth of Hawaiian green seaturtles (Chelonia mydas): an analysis based 908 on skeletochronology. - Fishery Bulletin 100: 117-127.

909

910 
912 Switch. To switch from juvenile to adult growth at $t_{\text {mat }}$ in (1), a logistic function is used:

$913 \quad S(t)=\frac{1}{1+e^{-k\left(t-t_{\mathrm{mat}}\right)}}$

914 where $k$ is any number large enough so that $\mathrm{S}(\mathrm{t})$ switches almost immediately from 0 to

9151 as soon as $t>t_{\text {mat }}$, thus approximating a Heaviside step function.

916 Reproductive investment. The reproductive investment $R(t+\Delta t)$ is given by the rate of

917 energy conversion to reproduction $c w(t)$ integrated over the period from $t$ to $t+\Delta t$,

918 expressed as a function of the somatic weights at the start $w(t)$ and at the end $w(t+\Delta t)$

919 of the time interval $\Delta t$, over which the reproductive events repeatedly occur. Assuming $920 \alpha=3 / 4$ and $\beta=\gamma=1$,

$R(t+\Delta t)=\int_{t}^{t+\Delta t} c w(\tau) d \tau=\int_{w(t)}^{w(t+\Delta t)} \frac{c}{a w^{\alpha}-(b+c) w} d w=\ldots$

$922=\frac{c}{b+c}\left[w_{t}-w_{t+\Delta t}+\frac{4 a}{3(b+c)}\left(w_{t}^{3 / 4}-w_{t+\Delta t}^{3 / 4}\right)+\frac{2 a^{2}}{(b+c)^{2}}\left(w_{t}^{1 / 2}-w_{t+\Delta t}^{1 / 2}\right)\right.$

$$
\left.+\frac{4 a^{3}}{(b+c)^{3}}\left(w_{t}^{1 / 4}-w_{t+\Delta t}^{1 / 4}\right)+\frac{4 a^{4}}{(b+c)^{4}}\left(\log \left(a-(b+c) w_{t}^{1 / 4}\right)-\log \left(a-(b+c) w_{t+\Delta t}^{1 / 4}\right)\right)\right]
$$

924 Code. A code example follows to illustrate the applied estimation method for one single

925 fish (object grodat). The weight scaling exponent $\alpha$ of energy acquisition rate, weight at 
927 boundaries for age at maturation $t_{m a t}$ and the asymptotic weight $w_{\infty}$ are species-specific.

928 The function indest runs the optimization (optimfun) over a grid of staring values,

929 removes aberrant estimates, returns the best fit and plots the fitted curve.

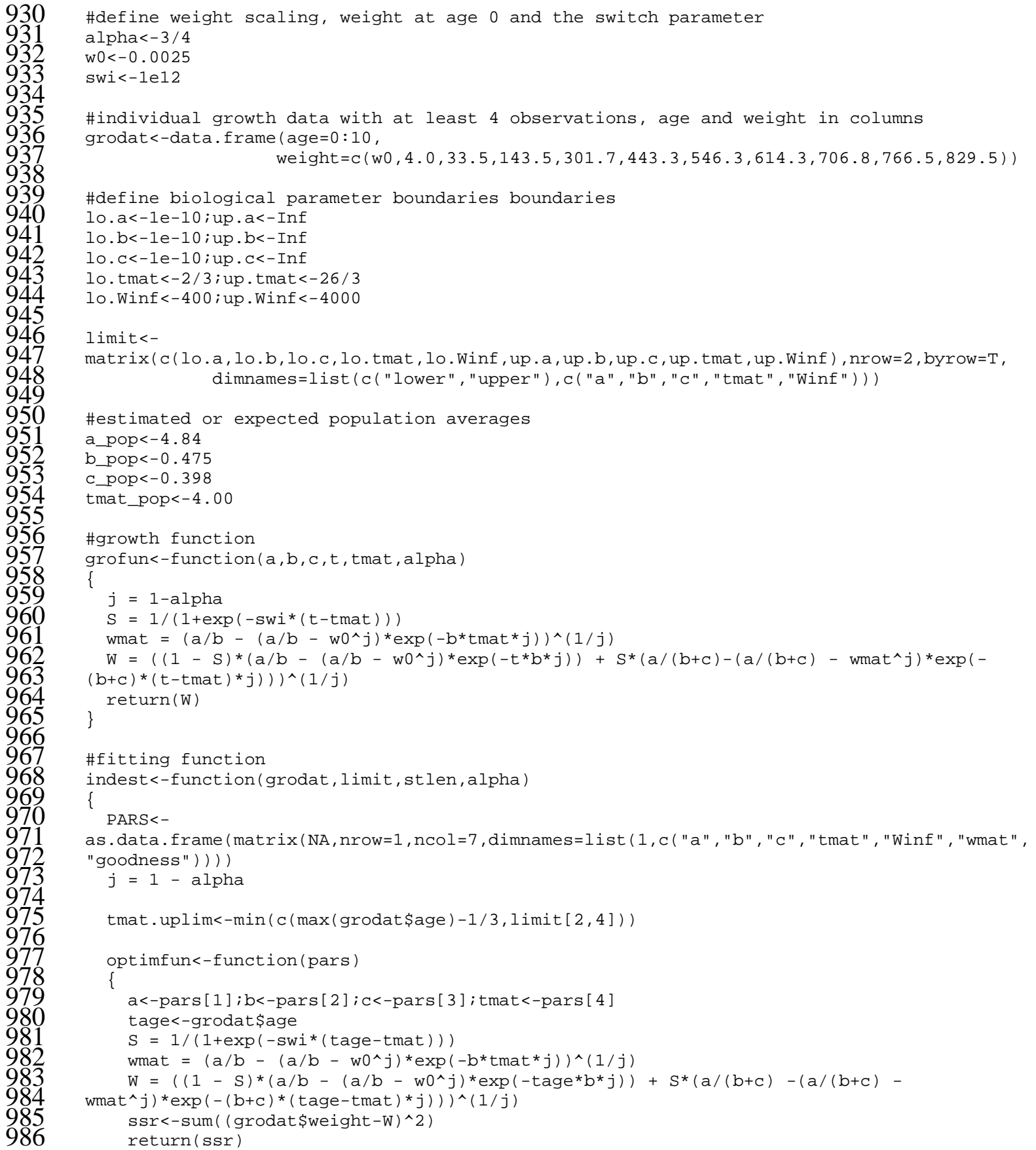




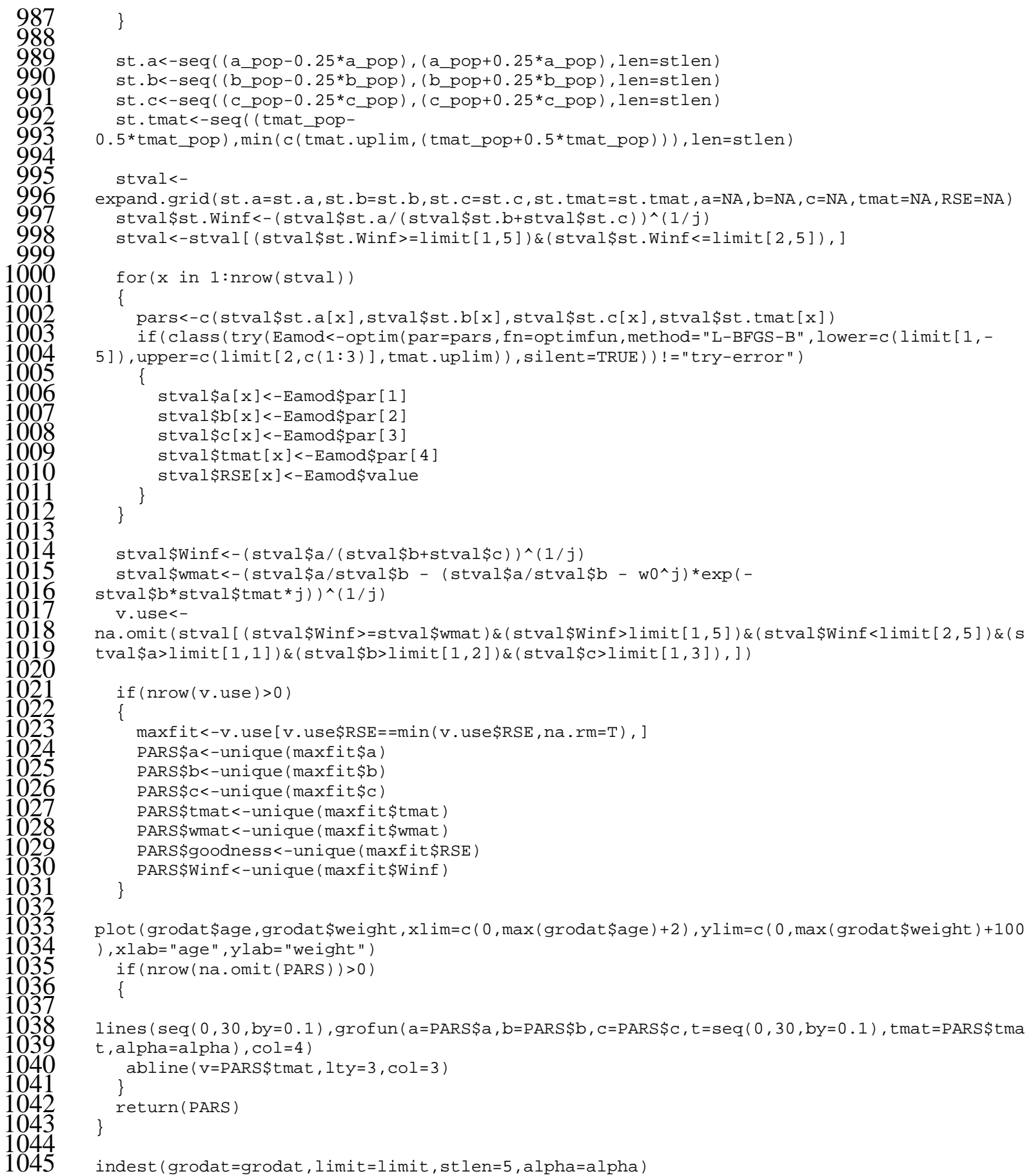


Table 1: Average of percentage bias $\mu \%$, coefficient of variation $C V$ and correlations $1047 r_{e}\left(x, x^{\prime}\right)$ between biases $e(x)$ and $e\left(x^{\prime}\right)$ in the estimates of the life history parameters $a$, $1048 b, c$ and $t_{\text {mat }}$ resulting from the four-trait estimation procedure applied to simulated data 1049 with (stochastic) and without (deterministic) environmental noise.

\begin{tabular}{|c|c|c|c|c|c|}
\hline & \multicolumn{5}{|c|}{ Deterministic } \\
\hline & $e(a)$ & $e(b)$ & $e(c)$ & $e(b+c)$ & $e\left(t_{\text {mat }}\right)$ \\
\hline$\mu \%$ & 0.00 & 0.01 & -0.02 & 0.00 & 0.00 \\
\hline$C V$ & 11.91 & 8.91 & 5.52 & 16.84 & 21.33 \\
\hline$r_{e}\left(a, x^{\prime}\right)$ & 1 & & & & \\
\hline$r_{e}\left(b, x^{\prime}\right)$ & 0.47 & 1 & & & \\
\hline$r_{e}\left(c, x^{\prime}\right)$ & -0.19 & -0.40 & 1 & & \\
\hline$r_{e}\left(b+c, x^{\prime}\right)$ & 0.36 & 0.18 & 0.53 & 1 & \\
\hline \multirow[t]{3}{*}{$r_{e}\left(t_{m a t}, x^{\prime}\right)$} & -0.09 & 0.60 & -0.07 & 0.17 & 1 \\
\hline & \multicolumn{5}{|c|}{ Stochastic } \\
\hline & $e(a)$ & $e(b)$ & $e(c)$ & $e(b+c)$ & $e\left(t_{\text {mat }}\right)$ \\
\hline$\mu \%$ & -0.15 & -0.32 & 0.23 & -0.18 & 0.30 \\
\hline$C V$ & 1.34 & 2.03 & 3.85 & 1.45 & 2.57 \\
\hline$r_{e}\left(a, x^{\prime}\right)$ & 1 & & & & \\
\hline$r_{e}\left(b, x^{\prime}\right)$ & 0.83 & 1 & & & \\
\hline$r_{e}\left(c, x^{\prime}\right)$ & -0.38 & -0.67 & 1 & & \\
\hline$r_{e}\left(b+c, x^{\prime}\right)$ & 0.94 & 0.76 & -0.21 & 1 & \\
\hline$r_{e}\left(t_{m a t}, x^{\prime}\right)$ & -0.07 & 0.17 & -0.27 & 0.00 & 1 \\
\hline
\end{tabular}


Table 2: Results of the regression analysis of the overall bias in life history parameters

1052 (Eq. 5) as a function of the potentially explanatory variables (Eq. 8) from a backward

1053 selection. Explanatory variables tested comprised of the coefficients of variation $C V_{a}$,

$1054 C V_{b}, C V_{c}$, the degree of autocorrelation $\theta_{a}, \theta_{b}, \theta_{c}$, and the correlations $r_{\text {sim }}(a, b)$,

$1055 r_{\text {sim }}(a, c), r_{\text {sim }}(b, c)$ of the simulated time series $a_{t}, b_{t}, c_{t}$, the age $t$ (i.e. the number of

1056 simulated data points) the number of experienced spawning events $y_{\text {mat }}$, the relative

1057 reproductive investment $q$, relative timing of onset of maturation $\tau$, and relative initial

1058 weight $v_{0}$.

\begin{tabular}{l||l|l||l|l||}
\multicolumn{2}{l||}{} & \multicolumn{2}{l||}{ four-trait estimation } & \multicolumn{2}{l||}{ three-trait estimation } \\
\hline \hline Selected variables & coefficient & p-value & coefficient & p-value \\
\hline \hline Intercept $\beta_{0}$ & 1.031 & $<10^{-3}$ & 0.475 & $<10^{-3}$ \\
\hline$C V_{a}$ & 0.418 & $<10^{-3}$ & 2.092 & $<10^{-3}$ \\
\hline$C V_{c}$ & - & - & -0.245 & $<10^{-3}$ \\
\hline$\theta_{a}$ & - & - & 0.032 & 0.087 \\
\hline$\theta_{c}$ & - & - & -0.026 & 0.066 \\
\hline$r_{\text {sim }}(a, b)$ & -0.066 & 0.002 & - & - \\
\hline$r_{\text {sim }}(a, c)$ & - & - & -0.085 & 0.001 \\
\hline age $t$ & -0.033 & $<10^{-3}$ & 0.014 & $<10^{-3}$ \\
\hline$y_{\text {mat }}$ & 0.044 & $<10^{-3}$ & - & - \\
\hline$q$ & -0.754 & $<10^{-3}$ & - & - \\
\hline$\tau$ & 0.047 & $<10^{-3}$ & -0.119 & $<10^{-3}$ \\
\hline$v_{0}$ & - & - & -30540 & 0.017 \\
\hline & & & & \\
\hline
\end{tabular}


1060 Table 3: Energy allocation parameters estimated for the1779 individual North Sea plaice 1061 growth trajectories using the four-trait and the three-trait model. The table gives the 1062 average $\mu$ and coefficient of variation $C V$, as well as the correlation coefficient $r\left(x, x^{\prime}\right)$ 1063 between the four life history parameters: energy acquisition $a$, maintenance $b$, 1064 reproductive investment $c$ and onset of maturation $t_{\text {mat }}$. For the four-trait estimation the 1065 results are displayed for only those estimations that belong to the second mode in the 1066 distribution of $b$ 's.

\begin{tabular}{|c|c|c|c|c|c|}
\hline & \multicolumn{5}{|c|}{ four-trait estimation: $2^{\text {nd }} b$-mode } \\
\hline & $a$ & $b$ & $c$ & $(b+c)$ & $t_{\text {mat }}$ \\
\hline$\mu$ & 5.31 & 0.57 & 0.32 & 0.90 & 4.45 \\
\hline$C V$ & 0.23 & 0.62 & 0.74 & 0.28 & 0.37 \\
\hline$r\left(a, x^{\prime}\right)$ & 1 & & & & \\
\hline$r\left(b, x^{\prime}\right)$ & 0.69 & 1 & & & \\
\hline$r\left(c, x^{\prime}\right)$ & -0.06 & -0.71 & 1 & & \\
\hline$r\left(b+c, x^{\prime}\right)$ & 0.93 & 0.74 & -0.06 & 1 & \\
\hline \multirow[t]{3}{*}{$r\left(t_{m a t}, x^{\prime}\right)$} & -0.22 & 0.30 & -0.63 & -0.18 & 1 \\
\hline & \multicolumn{5}{|c|}{ three-trait estimation: fixed $b$} \\
\hline & $a$ & $b$ & $c$ & $(b+c)$ & $t_{\text {mat }}$ \\
\hline $\bar{\mu}$ & 5.29 & 0.47 & 0.41 & 0.89 & 3.53 \\
\hline$\overline{C V}$ & 0.20 & - & 0.52 & 0.24 & 0.49 \\
\hline$r\left(a, x^{\prime}\right)$ & 1 & & & & \\
\hline$r\left(b, x^{\prime}\right)$ & - & - & & & \\
\hline$r\left(c, x^{\prime}\right)$ & 0.91 & - & 1 & & \\
\hline$r\left(b+c, x^{\prime}\right)$ & 0.91 & - & 1 & 1 & \\
\hline$r\left(t_{m a t}, x^{\prime}\right)$ & -0.68 & - & -0.64 & -0.64 & 1 \\
\hline
\end{tabular}


1067 Table 4: Estimated against true age at first maturity $A_{\text {mat }}$ in the 4- and three-trait 1068 estimation. The number of estimations falling in a true $A_{\text {mat }}$ class is given as a percentage 1069 of the total number of estimations in that true $A_{\text {mat }}$ class. The upper panel presents 1070 performances for age at maturation estimation by showing true against estimated $A_{\text {mat }}$ in 1071 the four-trait (simulated data set in which $a, b$ and $c$ vary stochastically), and the three1072 trait estimation (simulated data set in which $a$ and $c$ vary stochastically). Performance is 1073 slightly better for the three-trait estimation. Notice that the biases might not be 1074 representative for the real situation, since the simulated $C V$ 's might be higher than those 1075 applying in nature. The lower panel presents results of the application to real data by 1076 comparing the estimation of $A_{\text {mat }}$ between the four-trait and the three-trait estimation for 1077 both the entire data set and only the observations belonging to the $2^{\text {nd }} b$-mode. 1078 Agreement between the $t_{\text {mat }}$ estimates in the four- and three-trait estimation is very high 1079 and does not significantly change between the entire data set and the selected 1080 observations belonging to the $2^{\text {nd }} b$-mode. This indicates that the estimation of $t_{\text {mat }}$ or $1081 A_{\text {mat }}$ is not affected by confounding. 
1088

1089 1) Performance analysis

\begin{tabular}{|c|c|c|c|c|c|c|c|}
\hline $\begin{array}{l}\text { four-trait } \\
\text { simulated }\end{array}$ & \multicolumn{7}{|c|}{ True $A_{\text {mat }}$} \\
\hline \multirow{7}{*}{ 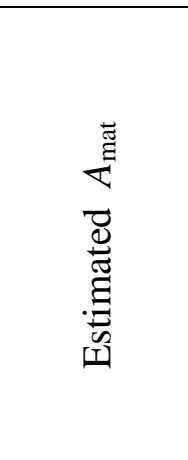 } & & 2 & 3 & 4 & 5 & 6 & 7 \\
\hline & 2 & 4 & 3 & 2 & 1 & 1 & 0 \\
\hline & 3 & 15 & 16 & 8 & 7 & 2 & 1 \\
\hline & 4 & 17 & 19 & 31 & 19 & 7 & 1 \\
\hline & 5 & 17 & 19 & 21 & 32 & 22 & 5 \\
\hline & 6 & 19 & 15 & 12 & 16 & 37 & 14 \\
\hline & 7 & 13 & 12 & 11 & 11 & 15 & 22 \\
\hline
\end{tabular}

\begin{tabular}{|c|c|c|c|c|c|c|c|}
\hline $\begin{array}{l}\text { three-trait } \\
\text { simulated }\end{array}$ & & \multicolumn{6}{|c|}{ True $A_{\text {mat }}$} \\
\hline \multirow{7}{*}{ 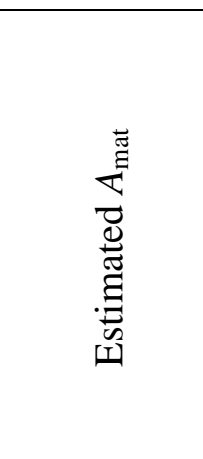 } & & 2 & 3 & 4 & 5 & 6 & 7 \\
\hline & 2 & 30 & 28 & 15 & 10 & 8 & 5 \\
\hline & 3 & 18 & 23 & 3 & 1 & 1 & 0 \\
\hline & 4 & 21 & 29 & 55 & 9 & 2 & 1 \\
\hline & 5 & 15 & 10 & 17 & 59 & 19 & 4 \\
\hline & 6 & 8 & 4 & 4 & 13 & 54 & 15 \\
\hline & 7 & 5 & 2 & 3 & 4 & 14 & 59 \\
\hline
\end{tabular}

1091

2) Application to real data

\begin{tabular}{|c|c|c|c|c|c|c|c|}
\hline $2^{\text {nd }} b$-mode & \multicolumn{7}{|c|}{ three-trait $A_{\text {mat }}$} \\
\hline \multirow{7}{*}{ 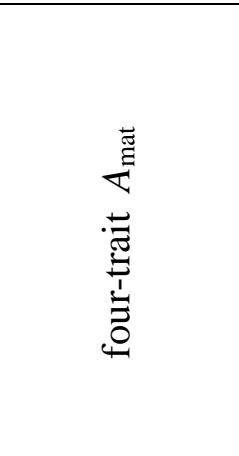 } & & 2 & 3 & 4 & 5 & 6 & 7 \\
\hline & 2 & 30 & 6 & 0 & 0 & 0 & 0 \\
\hline & 3 & 21 & 68 & 7 & 0 & 0 & 1 \\
\hline & 4 & 8 & 13 & 63 & 8 & 0 & 0 \\
\hline & 5 & 0 & 1 & 22 & 64 & 13 & 1 \\
\hline & 6 & 24 & 3 & 4 & 23 & 67 & 12 \\
\hline & 7 & 8 & 1 & 1 & 3 & 17 & 71 \\
\hline
\end{tabular}

\begin{tabular}{|c|c|c|c|c|c|c|c|}
\hline $\begin{array}{l}\text { All real data } \\
\text { observations }\end{array}$ & \multicolumn{7}{|c|}{ three-trait $A_{\text {mat }}$} \\
\hline \multirow{7}{*}{ 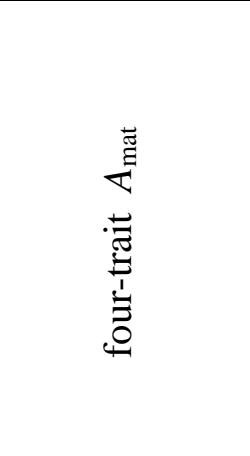 } & & 2 & 3 & 4 & 5 & 6 & 7 \\
\hline & 2 & 39 & 8 & 0 & 0 & 0 & 0 \\
\hline & 3 & 19 & 76 & 13 & 0 & 0 & 1 \\
\hline & 4 & 7 & 8 & 67 & 12 & 0 & 0 \\
\hline & 5 & 0 & 0 & 15 & 65 & 16 & 2 \\
\hline & 6 & 21 & 2 & 3 & 19 & 65 & 14 \\
\hline & 7 & 7 & 0 & 1 & 3 & 16 & 69 \\
\hline
\end{tabular}


1092 Figure 1: Density distributions of the four estimated parameters on real data. The first 1093 mode in the density distribution of maintenance $b$ (solid line) is likely an artifact due to 1094 confounding and corresponds to the second mode in the distribution of reproductive 1095 investment $c$. By selecting only observations belonging to the second mode fitted by a 1096 Gaussian mixture over parameter $b$, the first mode in the distribution of $b$ 's and the 1097 bump to the right in the distribution of $c$ 's are removed (dotted thick line).
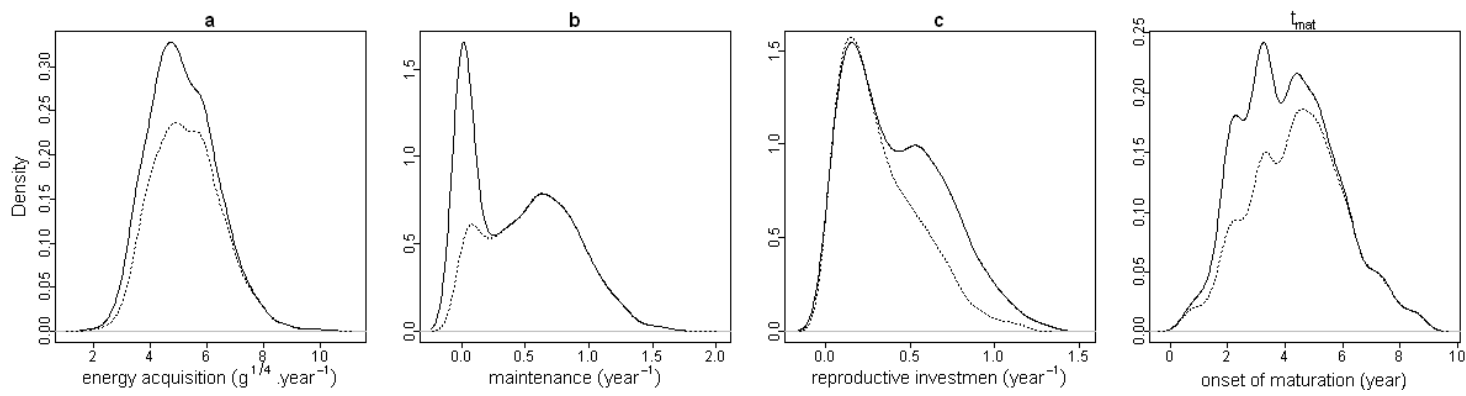
1099 Figure 2: Population fit of life-history on somatic size at age (solid lines) and estimated 1100 reproductive investment (dashed lines, see text). Error bars show 5\% and 95\% confidence 1101 intervals for the observations. For the gonads the averages of only mature fish are given 1102 whereas the fitted curve represents average population gonadic growth. The estimated life 1103 history parameters are $a=4.84 \mathrm{~g}^{1 / 4} \mathrm{yr}^{-1}, b=0.47 \mathrm{yr}^{-1}, c=0.40 \mathrm{yr}^{-1}, t_{\text {mat }}=4.00 \mathrm{yr}$.

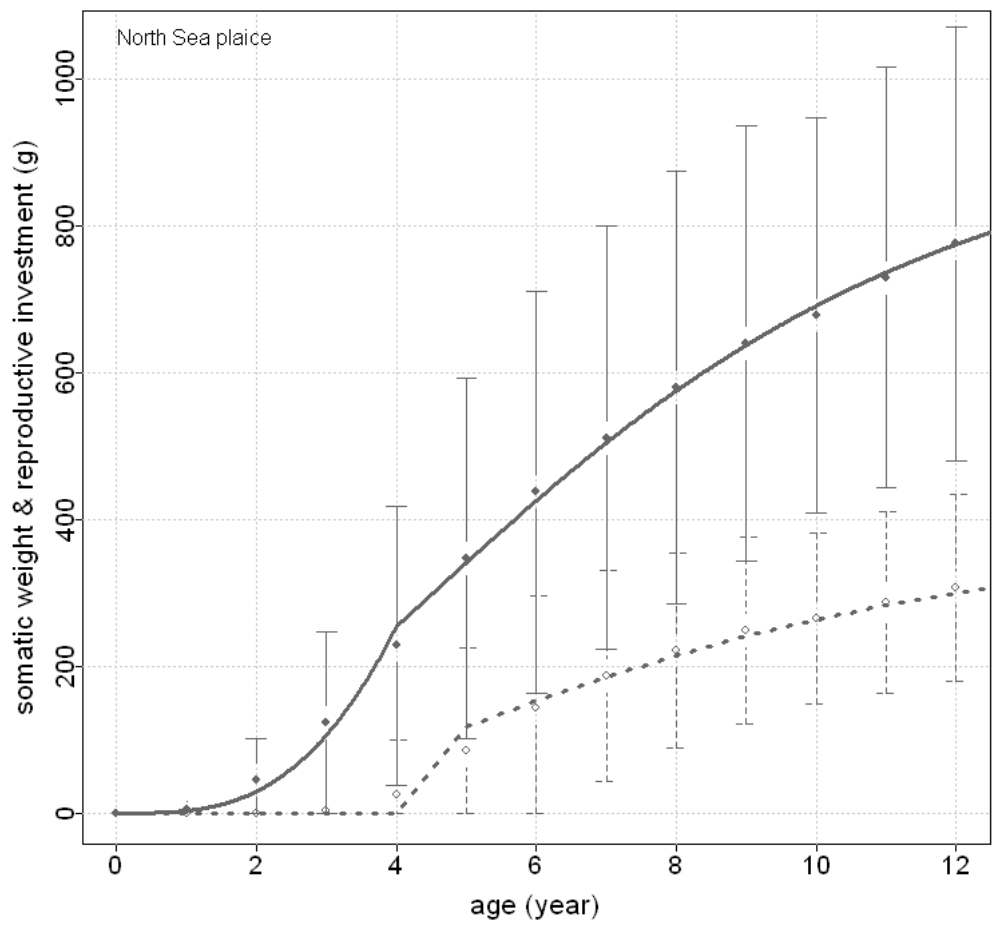

1104 
1105 Figure 3: Relationships of reproductive investment relative to size (RSI) and gonadic and 1106 migratory investment relative to total reproductive investment (GRI and MRI) as a 1107 function of size in the estimation of size-dependent reproductive investment. Because the 1108 probability of being mature depends also on age, the RSI slightly changes with age (see 1109 gray scale, the darker the older). The GRI has minimal contribution of $86 \%$ at a length of 1110 about $30 \mathrm{~cm}$ and increases thereafter. The cost of migration or MRI is accordingly 1111 maximal (14\%) at this size.
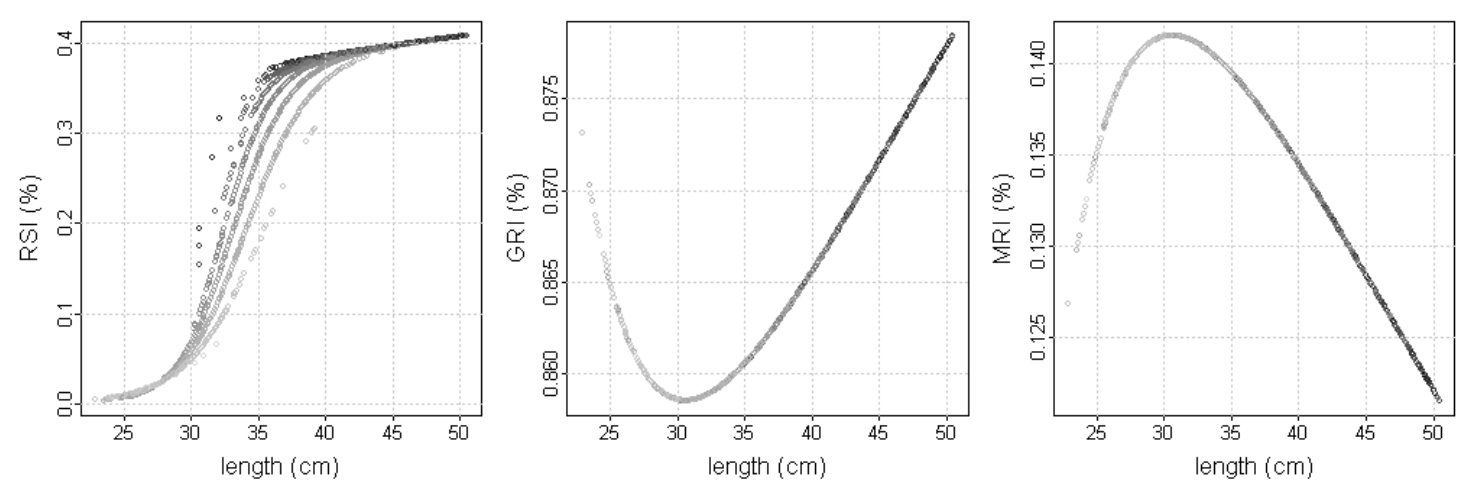

1112 
1113 Figure 4: Overall relative bias (Eq. 5) as a function of the true relative reproductive

1114 investment $q$, the true relative onset of maturation $\tau$, the true relative initial size $v_{0}$ and

1115 the number of years after the first spawning event $y_{\text {mat }}$ (rounded up $\left.\left(A_{\text {mat }}-t_{\text {mat }}\right)\right)$ in the

1116 deterministic case of the four-trait and the three-trait estimation. The simulation was

1117 based on all possible combinations for the observed ranges of the parameters: $a\{4,7\}$

$1118 \mathrm{~g}^{1 / 4} \mathrm{yr}^{-1}, b \quad\{0.4,0.9\} \mathrm{yr}^{-1}, c\{0.05,0.55\} \mathrm{yr}^{-1}$ and $t_{\text {mat }}\{1.25,5.25\}$ yr. Contours were

1119 obtained by fitting a non-parametric loess regression to the bias with span $=0.25$ for the

1120 two explanatory variables to be displayed. Bias becomes considerable if there are few

1121 observations $y_{\text {mat }}$ of the mature status, if the relative onset of maturation $\tau$ is very early

1122 and if the relative reproductive investment $q$ is small. Similar trends are found in the

1123 three-trait estimation but with lower relative biases and $q$ seems to have no more 1124 influence on the bias. 

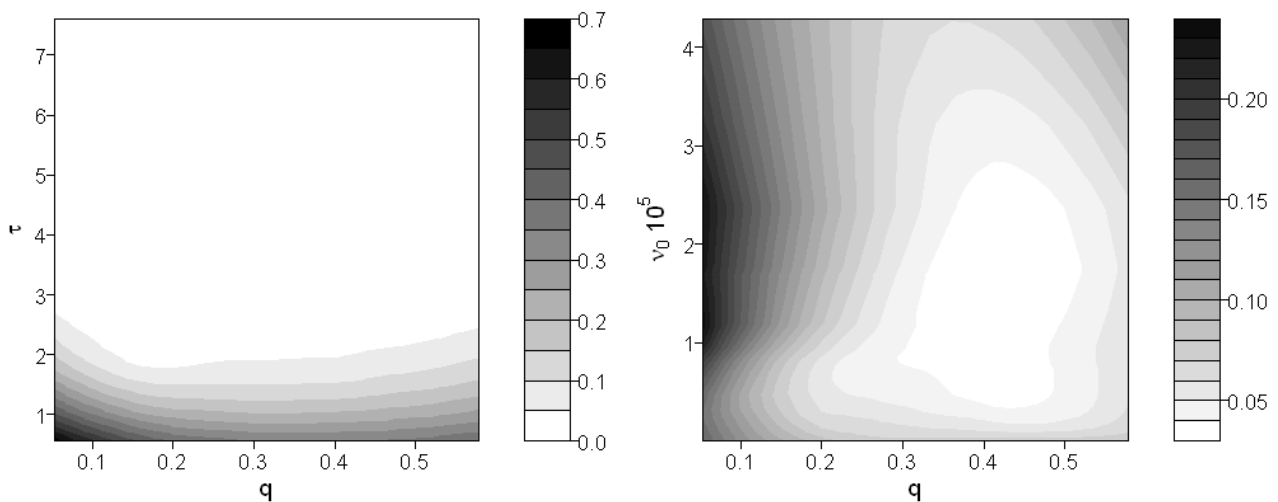

1129
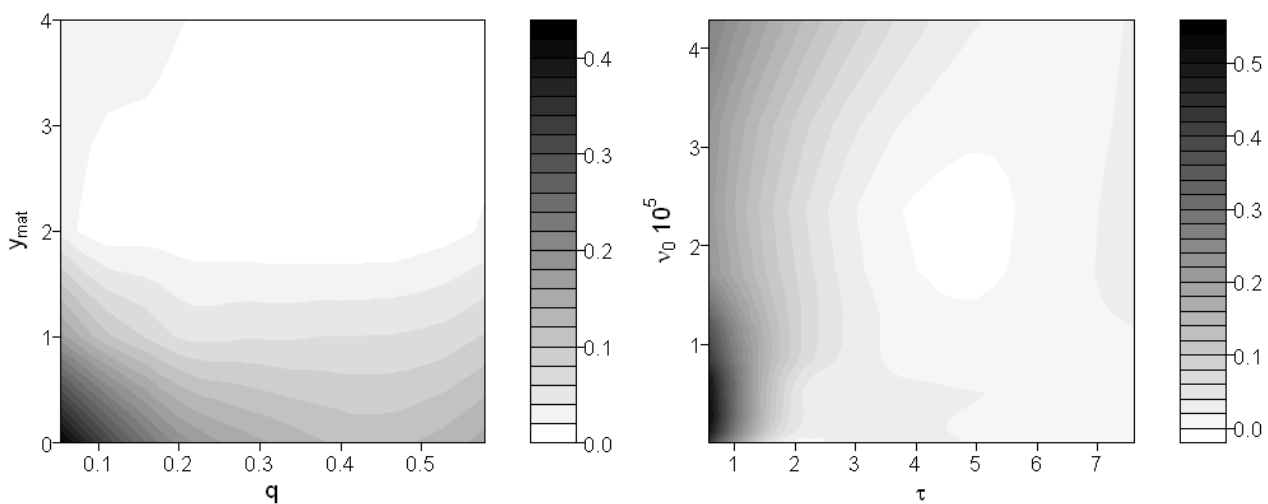

1130
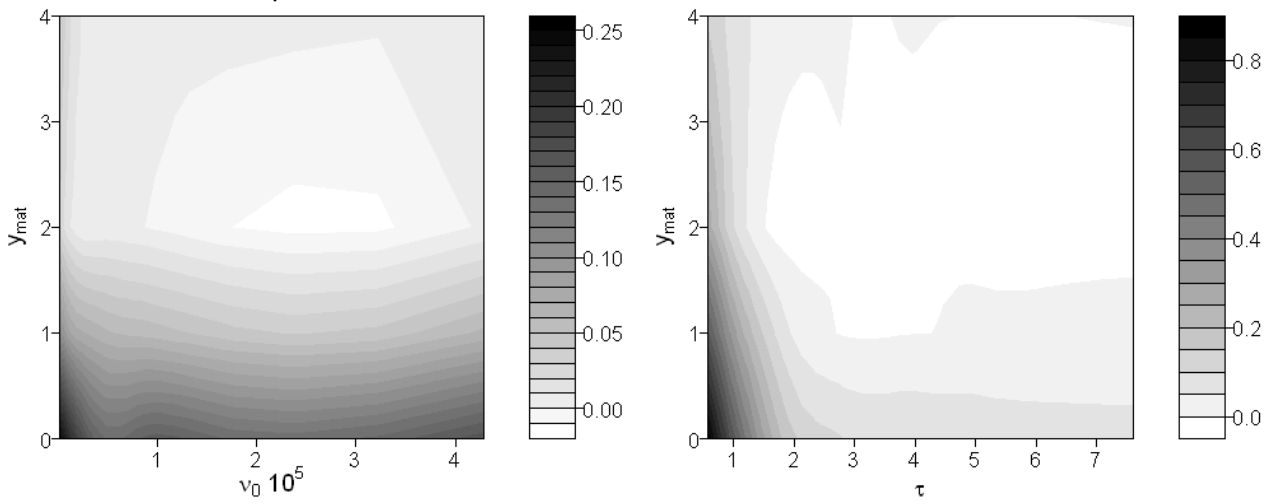
1132

1133
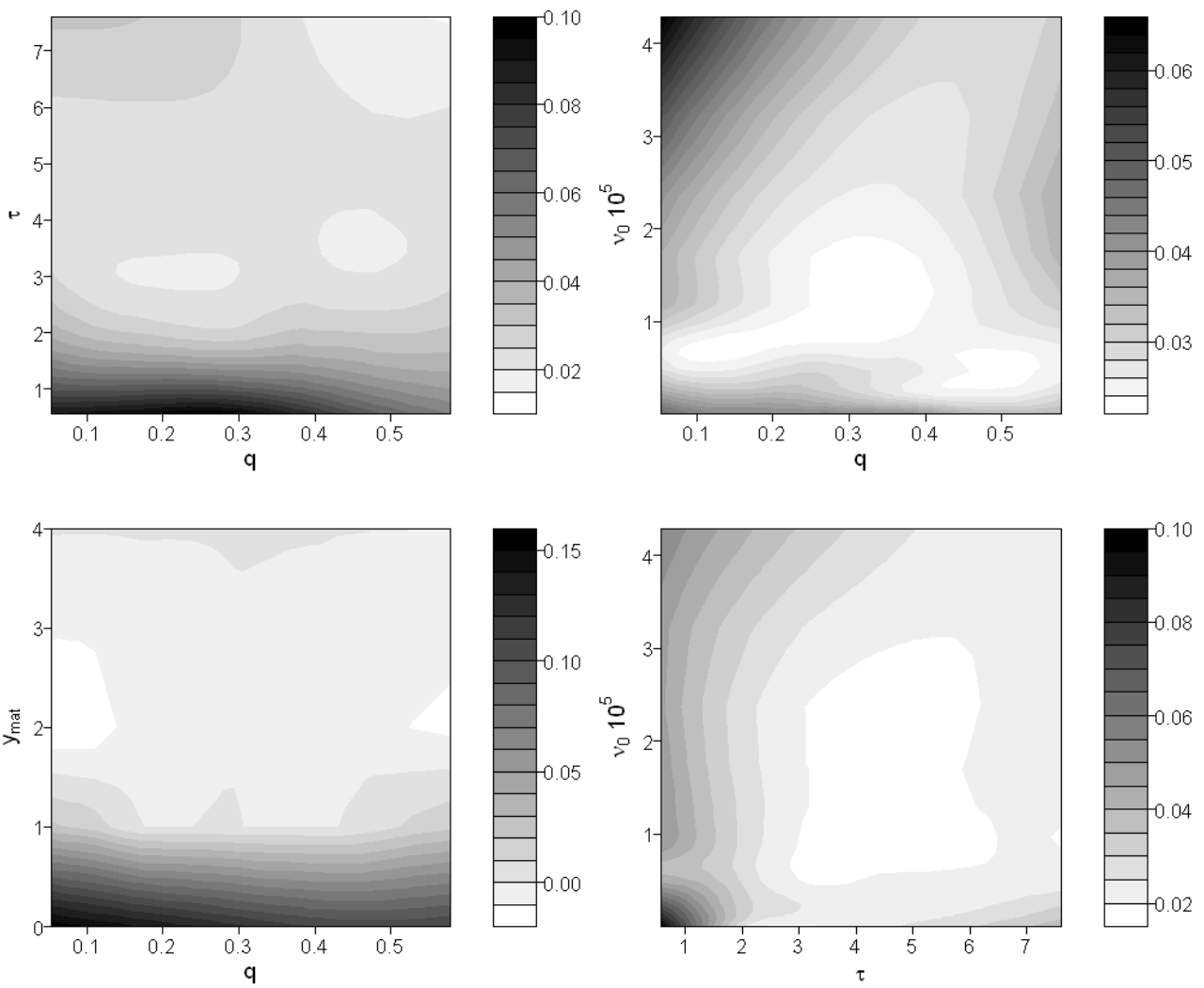

1134
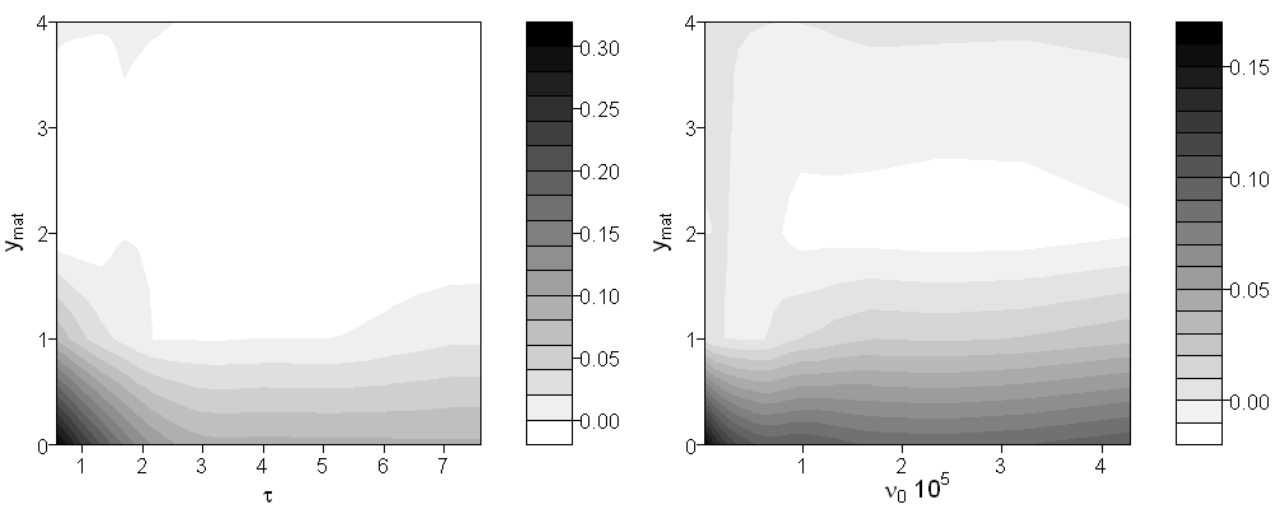

1135 
1136 Figure 5: Density distributions of the four estimated life history parameters and 1137 relationships between parameter biases estimated on simulated data with environmental 1138 noise. Very similar parameter distributions as from real data (see Figure 1) are obtained 1139 in the simulation (first row), in which the covariance structure from the selected 1140 distribution modes from real data was used. The regressions between parameter biases 1141 (dashed lines) show that the biases of $b$ and $c$ are negatively correlated, whereas the bias 1142 of $(b+c)$ is on average smaller than bias of each of its components. The strong positive 1143 correlation between $a$ and $(b+c)$ is a consequence of fitting to an asymptotic size: the 1144 higher $a$ is, the higher $(b+c)$ has to be to reach the same asymptotic size. The same 1145 effect translates to $b$ but not to $c$.

1146 
1157
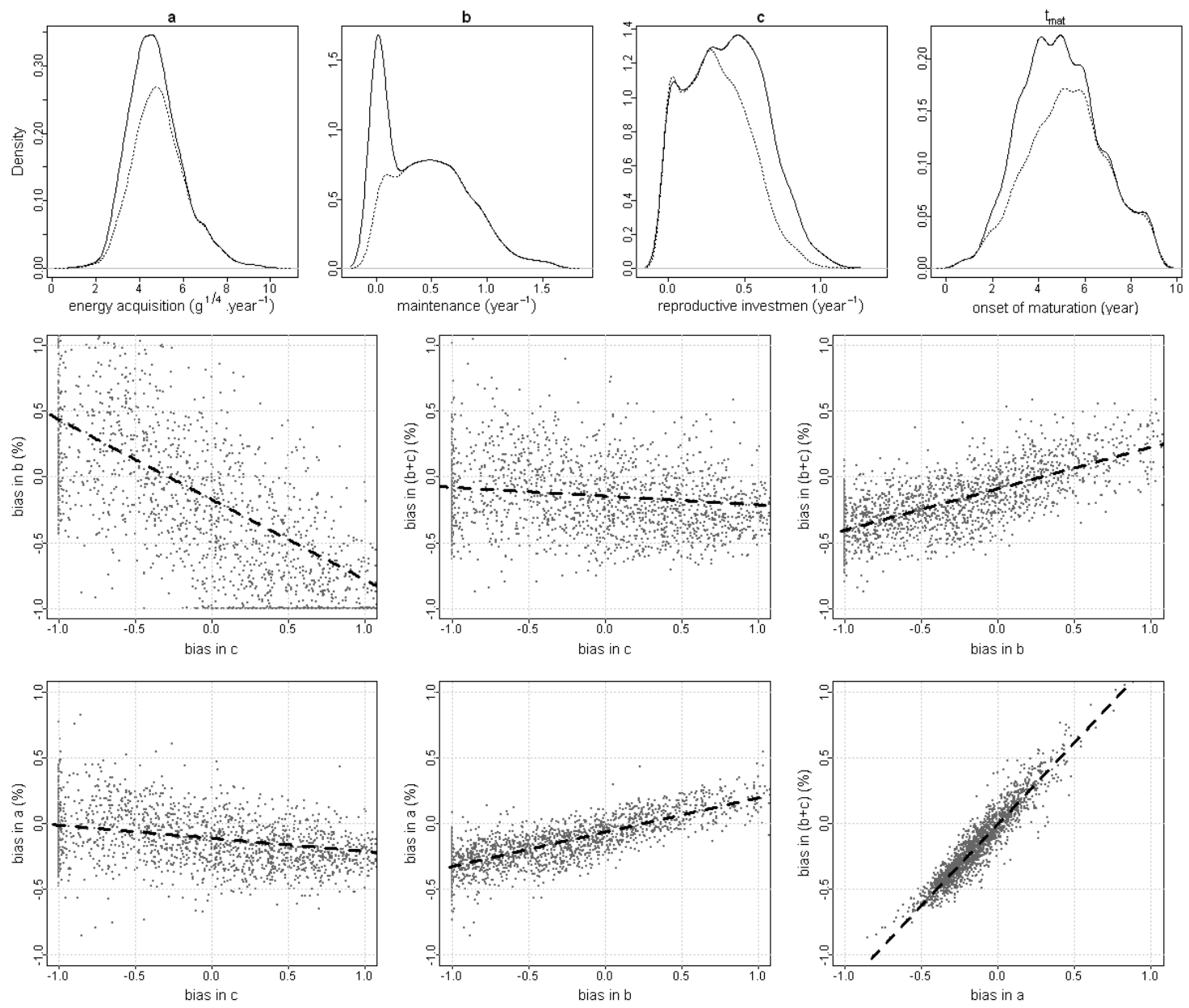

1158 
1159 Figure 6: Relative biases in $a, b, c$ and $t_{\text {mat }}$ in the four-trait estimation and $a, c$ and $1160 t_{\text {mat }}$ in the three-trait estimation, resulting from environmental variation, shown as a 1161 function of the $C V$ in the simulated time series of $a, b$, and $c$ (four-trait estimation) or $1162 a$ and $c$ (three-trait estimation). The estimated parameters are given relative to the 1163 geometric mean of the time series of $a, b$, and $c$. The $C V$ is given by the geometric 1164 mean of the realized $C V$ 's in series of $a, b$ and $c$. Black lines show a quantile 1165 regression through these biases for the 50\% (dashed line) and the 5\% and 95\% quantiles 1166 (dotted lines). Notice that the biases are strictly evaluated only for $t_{\text {mat }}$, since the true 1167 reference values of the varying $a, b$ and $c$ is not really known. Furthermore the 1168 simulated $C V$ 's might be higher than those applying in nature. 

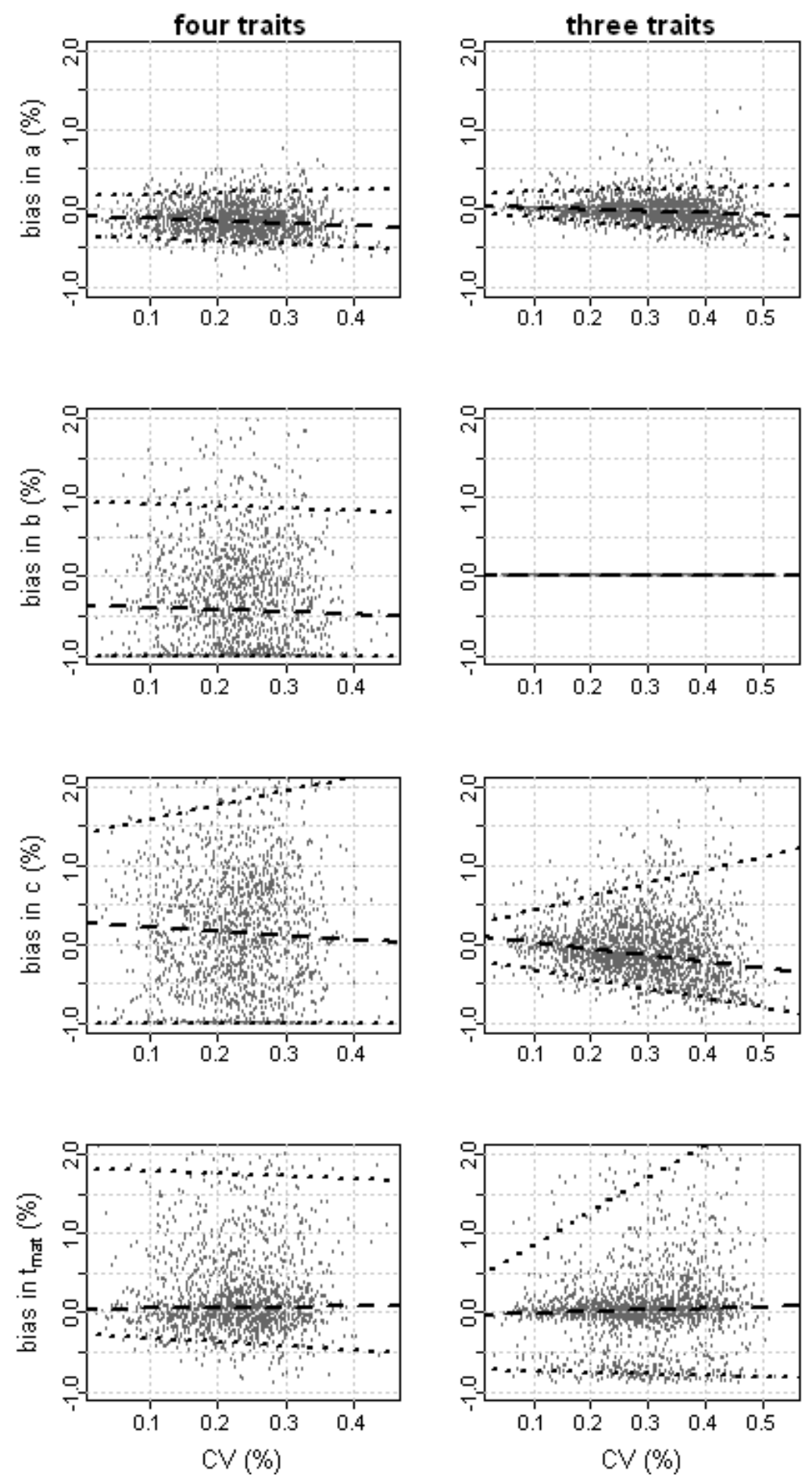
1171 Figure 7: Sensitivity of the parameters estimates $a, b, c$ and $t_{\text {mat }}$ to an incorrect

1172 assumption about the allometric scaling exponent $\alpha\left(\alpha_{\text {sim }}=3 / 4\right.$ whereas $\alpha_{\text {fit }}=2 / 3$ or

$\left.1173 \alpha_{\text {fit }}=4 / 5\right)$ in the four- and the three-trait estimation. It was accounted for that different

1174 allometric scaling exponents would result in different assumptions about the constant

1175 maintenance by fitting the energy allocation model to the population growth curve

$1176 \quad\left(b_{\alpha=2 / 3}=0.175\right.$ year $^{-1}, b_{\alpha=3 / 4}=0 . .459$ year $^{-1}, b_{\alpha=4 / 5}=0.864$ year $^{-1}$, leading to different

1177 solutions of Eq. 4). The estimated against the true parameters are shown, black dots

1178 representing the estimates assuming the correct allometric scaling exponent $(\alpha=3 / 4)$,

1179 typically on the $45^{\circ}$-line, light gray "“-” and dark gray "+" represent the estimates by

1180 assuming falsely a too low $(\alpha=2 / 3)$ or too high $(\alpha=4 / 5)$ scaling exponent

1181 respectively, whereas the light grey and dark gray dotted lines represent the regression

1182 through these estimated and true data points assuming wrong scaling.

1183 

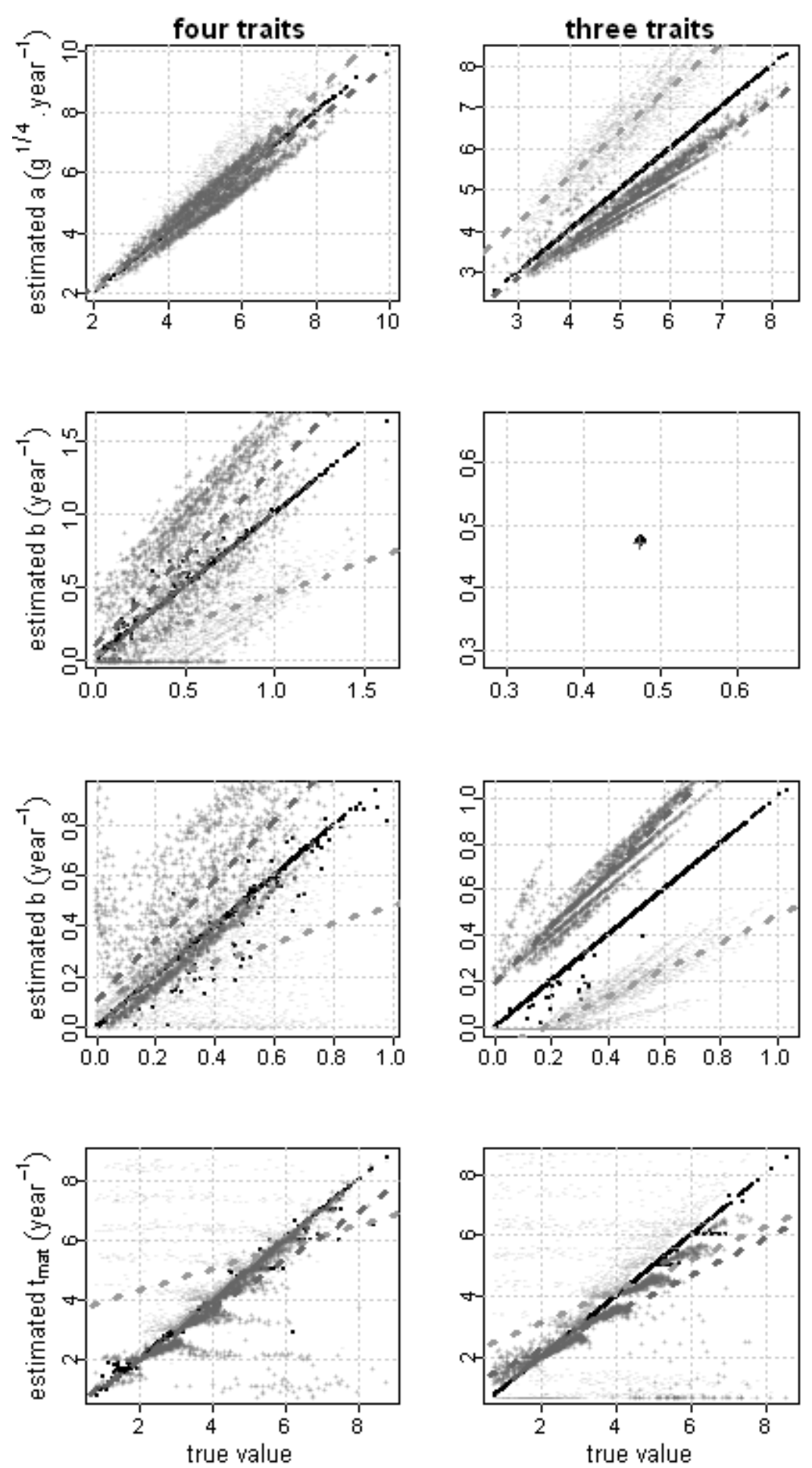

1184 
1185 Figure 8: Comparison of reaction norms derived from the 3 trait estimation of individual

1186 life history in this study (gray lines) with reaction norm estimated by from Grift, et al.

1187 (2003) averaged over the past 5 decades by only using cohorts for which more than 30

1188 observations were available. Dotted lines represent the $25 \%$ - and $75 \%$ probabilities of

1189 maturation, the dashed line represents the average length at age. The reaction norm from

1190 individual life history estimation is shown for an interpretation of the first spawning

1191 event $A_{\text {mat }}$ given by $t_{\text {mat }}$ plus a minimal period of preparation for spawning of 4 months,

1192 rounded up to the next year.

1193

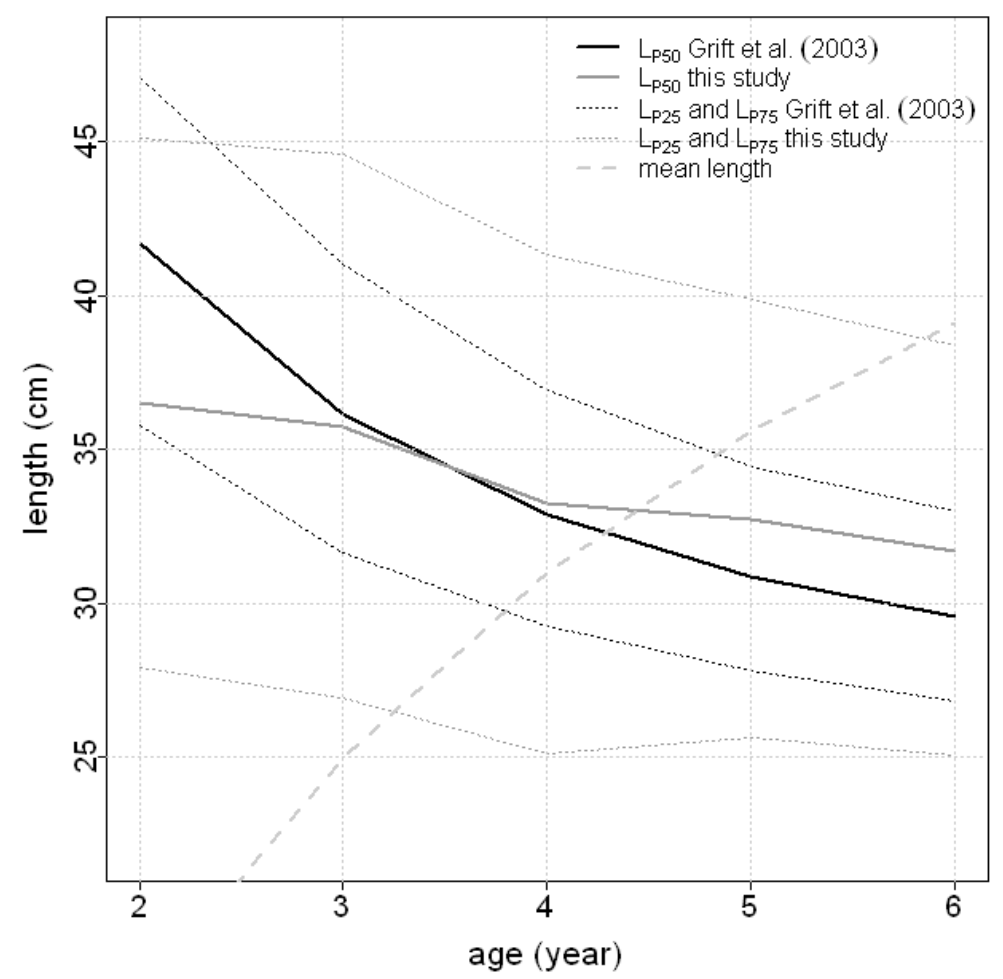

1194 